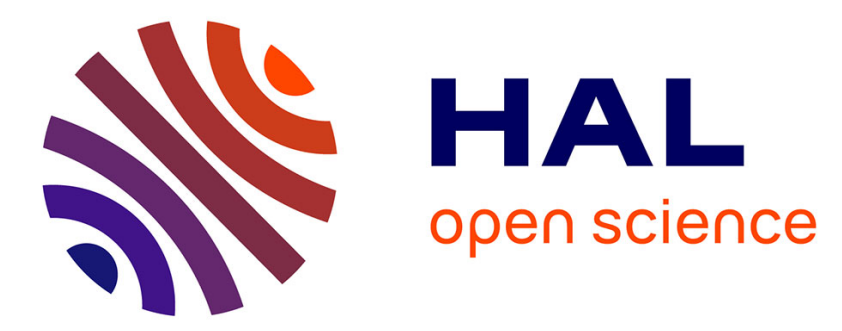

\title{
Experimental study of the stabilization of Tollmien-Schlichting waves by finite amplitude streaks
}

\author{
J.H.M. Fransson, L. Brandt, A. Talamelli, Carlo Cossu
}

\section{To cite this version:}

J.H.M. Fransson, L. Brandt, A. Talamelli, Carlo Cossu. Experimental study of the stabilization of Tollmien-Schlichting waves by finite amplitude streaks. Physics of Fluids, 2005, 17 (5), pp.054110054110-15. 10.1063/1.1897377 . hal-01023377

\section{HAL Id: hal-01023377 \\ https://hal-polytechnique.archives-ouvertes.fr/hal-01023377}

Submitted on 28 Jul 2014

HAL is a multi-disciplinary open access archive for the deposit and dissemination of scientific research documents, whether they are published or not. The documents may come from teaching and research institutions in France or abroad, or from public or private research centers.
L'archive ouverte pluridisciplinaire HAL, est destinée au dépôt et à la diffusion de documents scientifiques de niveau recherche, publiés ou non, émanant des établissements d'enseignement et de recherche français ou étrangers, des laboratoires publics ou privés. 


\section{AIP | Physics of Fluids}

\section{Experimental study of the stabilization of Tollmien-Schlichting waves by finite amplitude streaks}

Jens H. M. Fransson, Luca Brandt, Alessandro Talamelli, and Carlo Cossu

Citation: Physics of Fluids (1994-present) 17, 054110 (2005); doi: 10.1063/1.1897377

View online: $\mathrm{http}: / / \mathrm{dx}$.doi.org/10.1063/1.1897377

View Table of Contents: http://scitation.aip.org/content/aip/journal/pof2/17/5?ver=pdfcov

Published by the AIP Publishing

\section{Articles you may be interested in}

Compressible laminar streaks with wall suction

Phys. Fluids 25, 054110 (2013); 10.1063/1.4807066

Detuned resonances of Tollmien-Schlichting waves in an airfoil boundary layer: Experiment, theory, and direct numerical simulation

Phys. Fluids 24, 094103 (2012); 10.1063/1.4751246

Wall heat transfer effects on Klebanoff modes and Tollmien-Schlichting waves in a compressible boundary layer Phys. Fluids 21, 024106 (2009); 10.1063/1.3054155

The stabilizing effect of streaks on Tollmien-Schlichting and oblique waves: A parametric study Phys. Fluids 19, 078103 (2007); 10.1063/1.2746047

Interaction between Görtler vortices and two-dimensional Tollmien-Schlichting waves

Phys. Fluids 12, 1461 (2000); 10.1063/1.870395

\section{A|P| Journal of}

Journal of Applied Physics is pleased to announce André Anders as its new Editor-in-Chief 


\title{
Experimental study of the stabilization of Tollmien-Schlichting waves by finite amplitude streaks
}

\author{
Jens H. M. Fransson and Luca Brandt \\ KTH Mechanics, SE-100 44 Stockholm, Sweden \\ Alessandro Talamelli \\ KTH Mechanics, SE-100 44 Stockholm, Sweden and II Facoltà di Ingegneria, Università di Bologna, \\ 47100 Forlì, Italy \\ Carlo Cossu \\ LadHyX, CNRS-Ecole Polytechnique, F-91128 Palaiseau, France
}

(Received 11 November 2004; accepted 24 February 2005; published online 3 May 2005)

\begin{abstract}
It has recently been found by using temporal and spatial numerical simulations that steady optimal streaks of moderate amplitude, i.e., sufficiently large but not exceeding the critical amplitude for the inflectional instability, are able to reduce the growth of Tollmien-Schlichting (TS) waves up to their complete suppression. This investigation aims at experimentally verifying this stabilizing effect by generating stable and symmetric, close to sinusoidal, streaks of moderate amplitudes $(\sim 12 \%$ of the free-stream velocity) by means of a spanwise array of cylindrical roughness elements. The three-dimensional (3D) streaky base flow is then subjected to a secondary instability generated through a spanwise slot in the plate by means of regulated blowing and suction. In this study the stabilizing role of the streaks on TS waves is unambiguously confirmed and by increasing the height of the roughness elements, thus inducing larger amplitude streaks, we are also able to show that the stabilizing action on the TS waves increases with the streak amplitude. These results are the first to confirm the numerical predictions reported in earlier works. The full cross-stream plane has been measured at different downstream positions allowing a complete evaluation and comparison of the different amplitude measures used in previous experimental works. Furthermore, theoretical impulse response analysis and stability calculations are applied to the present experimental streaky base flow enabling a qualitative comparison of the 3D modulated TS wave distribution. (C) 2005 American Institute of Physics. [DOI: 10.1063/1.1897377]
\end{abstract}

\section{INTRODUCTION}

\section{A. Boundary layer streaks}

The boundary layer developing on a flat plate is nominally two dimensional (2D) and is well described by the Blasius and Falkner-Skan similarity solutions when the pressure gradient is constant along the plate. ${ }^{1}$ However, it has been known for a long time that it is very difficult to observe a two-dimensional (2D) boundary layer unless in extremely well controlled situations. Very small amounts of noise, such as free-stream turbulence or wall imperfections, are able to induce non-negligible spanwise variations of the boundary layer profiles. This sensitivity is due to the "lift-up" effect: ${ }^{2}$ streamwise vortices of very low amplitude, living in a high shear region such as the boundary layer, are able to mix very efficiently low momentum and high momentum fluid. This eventually leads to large elongated spanwise modulations of the streamwise velocity field called streamwise streaks. The input/output energy ratio of the process has been optimized in the linear framework ${ }^{3,4}$ allowing the computation of the optimal perturbation. This perturbation takes the form of streamwise vortices, which induce steady and spanwise periodic streamwise streaks once forced at the leading edge of the flat plate.

\section{B. Previous investigations of the stability of streaky boundary layers}

\section{Theoretical and numerical findings}

In the absence of streaks, the 2D boundary layer becomes linearly unstable as the Reynolds number exceeds a critical value; the instability is of viscous nature and the first amplified wave is the 2D Tollmien-Schlichting (TS) wave. However, the linear stability property changes in the presence of streaks. There is now a consensus that inflectional type, high frequency, linear instabilities of inviscid nature develop if the streak amplitude is large enough. A "numerical" family of streaky boundary layers has been obtained in Ref. 5 by forcing steady optimal vortices ${ }^{3,4}$ of finite amplitude at the flat plate leading edge and by then computing the induced flow by fully nonlinear simulations. By increasing the amplitude of the vortices, streaky boundary layer flows of increasing maximum streak amplitude are obtained. For this particular but well defined family, the inflectional instability sets in when the amplitude of the streaks $A_{\mathrm{ST}}$ is larger than $26 \%$ of the free-stream velocity $U_{\infty}$ in the inviscid limit, ${ }^{5}$ it is of sinuous antisymmetric type and of convective nature. ${ }^{6}$

The viscous linear stability of the streaks of the same family but of moderate amplitude, $A_{\mathrm{ST}}<0.26 U_{\infty}$, and therefore stable to inflectional instabilities, has later been investi- 
gated both by direct numerical simulation ${ }^{7}$ and linear stability analysis. ${ }^{8}$ It was found that in the presence of the streaks, the most unstable viscous waves evolve from 2D-TS waves into three-dimensional (3D) varicose fundamental modes, here called "streaky-TS waves," having almost identical phase speed but lower growth rates than the corresponding 2D-TS waves. The stabilizing effect of the streaks on the TS waves was found to increase with the streak amplitude and begins to be non-negligible for streaks of amplitudes larger than about $8 \%$ of $U_{\infty}$. The comparison of these findings with previous numerical and experimental studies has not been straightforward because, in the past, most of the attention has been focused on possible destabilizing resonances between finite amplitude TS waves and streaks of the same order of magnitude (about $1 \%$ of $U_{\infty}$ ). Here we are interested in the different case in which the streaks have already reached moderate amplitudes, larger than about $5 \%$ of $U_{\infty}$, and TS waves are of very small amplitudes, smaller than $0.5 \%$ of $U_{\infty}$. Therefore we consider the streaky boundary layer as a 3D base flow in which linear 3D waves develop. This approach is appropriate, for instance, in the case if one would artificially force streaks of finite amplitude in order to delay the instability of the TS waves. In this context, only a few investigations, essentially experimental are currently available, as summarized below, and when seen together they give a somehow puzzled picture.

\section{Experimental findings}

A first type of streaky boundary layers is where streaks are randomly forced by free-stream turbulence ${ }^{9-11}$ where "natural" TS waves were detected. ${ }^{12}$ TS waves forced with a vibrating ribbon in a boundary layer subject to free-stream turbulence were found to be less amplified than the corresponding 2D-TS waves but traveled at a very similar phase speed. ${ }^{13}$ The unsteadiness of the streaks as well as the random character of their oscillations make the detailed comparison of these results with those from the linear stability of the steady optimal streaks difficult.

Steady streaks have been generated using different experimental techniques resulting in different streak shapes. The family of streaky boundary layers presumably most resembling the numerical family induced by optimal vortices was obtained by Tani and Komoda. ${ }^{14}$ These authors used small wings located outside the boundary layer to generate the steady streamwise vortices. The maximum streak amplitude achieved in these experiments was about $7 \%$ of $U_{\infty}$. TS waves were then forced in the boundary layer and they showed characteristic M-shaped wall-normal profiles. However, no explicit measure of the growth rates or of the phase speeds of such waves was provided. Kachanov and Tararykin ${ }^{15}$ generated the streaks by means of blowing and suction at the wall. The maximum streak amplitude was reached in this case at the location of the blowing-suction slot. The forced TS-like waves had essentially the same M-shaped structure previously observed by Tani and Komoda. ${ }^{14}$ These 3D waves were seen to travel at essentially the same phase speed of the corresponding 2D-TS waves but they were less amplified. In this study, the TS wave amplitude was computed by spanwise integration of the rms values taken at a fixed distance from the wall.

Bakchinov and co-workers ${ }^{16}$ generated the streaks by using a spanwise periodic array of roughness elements placed at the wall. For moderate streak amplitudes $\left(\sim 15 \%\right.$ of $\left.U_{\infty}\right)$ where, no high frequency (inviscid-type) instabilities are present, TS-like traveling waves are measured, with shapes in accordance with the previous studies mentioned above. ${ }^{14,15}$ Taking as a measure of their amplitude the wallnormal maximum of the rms profiles at different spanwise stations, the waves were seen to grow in the high-speed streak and decay in the low-speed streak.

Note that all three studies above show that the disturbance amplitude appears to grow faster in the high-speed region as compared to the low-speed region, which is in contrast to what intuitively is expected by considering only the stability of the local velocity profile.

\section{Scope of the present investigation}

Even if the available experimental data on the shape and phase speed of the TS waves developing in the presence of streaks are in qualitative agreement and may allow for a comparison with the numerical studies by Cossu and Brandt, ${ }^{7,8}$ there is still no conclusive experimental confirmation of the predicted stabilizing effect of the streaks. As the base flow (the streaks) is 3D and changes in the streamwise direction it is likely that the corresponding 3D TS waves will also change their shape while traveling downstream. In that case the best measure of their amplitude at a given streamwise station is probably given by an integration of the rms values over the cross-stream (wall-normal/spanwise) plane. In particular, the local energy density (over the cross-stream plane) measure was used in Refs. 7 and 8. However, in the cited experimental studies the amplitude of the TS waves was computed at fixed spanwise or wall-normal positions. This leaves some uncertainty on whether the observed downstream evolution of the amplitude depends on a change in the energy of the wave or on a relevant deformation of their shape at constant energy. Furthermore the experimentally generated base flow were not detailed enough to allow for a comparison with the numerical findings obtained on the very idealized family of optimal streaks. It is therefore impossible to assess whether the behavior of these waves is due to the different amplitude of the underlying streaks, due to their particular shape, or due to the particular measure chosen to quantify the traveling wave's amplitude.

The main motivation for the present study has therefore been to provide full cross-plane experimental measures of the downstream development of 3D TS-like waves in well documented streaky boundary layers. These data would allow to compare the different measures adopted for the wave amplitude and to conclude about the stabilizing role of the streaks for the particular flow under consideration. The present streaks are generated by a spanwise array of equally spaced roughness elements placed at the wall inside the boundary layer at a given downstream distance from the 


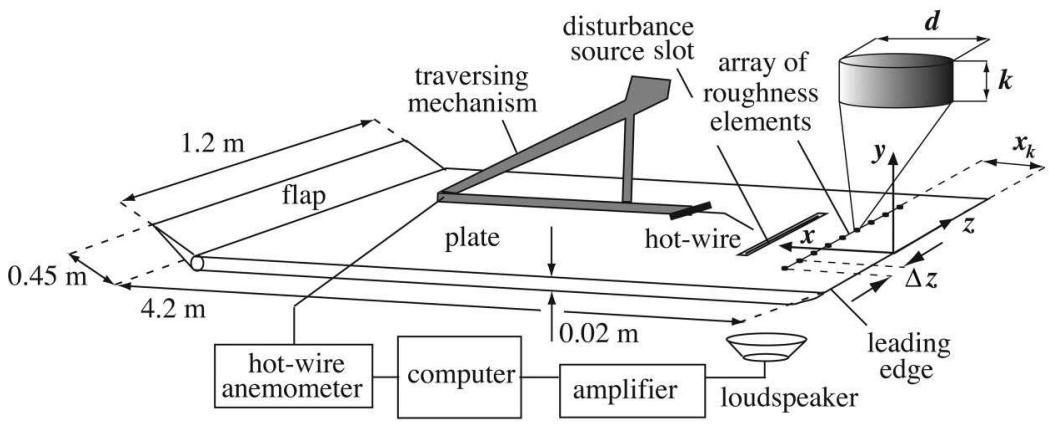

FIG. 1. Schematic of the setup. $k=1.3$ or $1.4 \mathrm{~mm}, d$ $=4.2 \mathrm{~mm}, \Delta z=14.7 \mathrm{~mm}$, and $x_{k}=80 \mathrm{~mm}$. leading edge. The characteristics of the resulting streaky boundary layer have been investigated and fully documented in a previous study. ${ }^{17}$

The paper is organized as follows. In Sec. II the experimental configuration used to generate the streaky base flows and force the time periodic TS waves is presented. In Sec. III, the essential features of the boundary layer flows, in which the TS waves are introduced, are described. The amplitude evolution and the shape of the 3D streaky TS waves is fully documented in Sec. IV, where the stabilizing role of the streaks is unambiguously confirmed. In this section the effect of choosing different amplitude measures is also discussed, followed by some theoretical comparisons and phase speed calculations. The paper ends with a summary of the main conclusions.

\section{EXPERIMENTAL SETUP AND EXTERNAL PRESSURE GRADIENT}

\section{A. Test facility}

The experiments were carried out at KTH Mechanics in the minimum-turbulence-level (MTL) wind tunnel with a setup similar to the one used in Ref. 17. A $4.2 \mathrm{~m}$ long flat plate was mounted horizontally in the test section whose dimensions are $7 \mathrm{~m}$ in length, $0.8 \mathrm{~m}$ in height, and $1.2 \mathrm{~m}$ in width. The high flow quality of the MTL tunnel was recently reconfirmed. At $25 \mathrm{~ms}^{-1}$ the streamwise turbulence intensity is less than $0.025 \%$ of $U_{\infty}$ while both the cross flow turbulence intensities are less than $0.035 \%$. More details can be found in an internal technical report by Lindgren and Johansson. $^{18}$

Figure 1 shows a schematic of the experimental setup. The plate was the same as described in Klingmann et al. ${ }^{19}$ designed in order to get a short pressure gradient region without any suction peak at the leading edge region (cf. the work by Fransson ${ }^{20}$ ). A trailing edge flap is also mounted in order to compensate for the extra blockage below the plate due to the supports and the piping system for the TS wave generation.

A Cartesian coordinate system is introduced (cf. Fig. 1). The origin is located on the centerline at the leading edge with $x, y$, and $z$ axis directed along the streamwise, wallnormal, and spanwise direction, respectively.

A single hot-wire probe, mounted on a computer controlled five degree of freedom traversing mechanism, was used to measure the streamwise velocity component (here denoted by $U$ ) using an AN-1003 constant temperature an- emometer. The probe was built at KTH Mechanics and was made of a $2.5 \mu \mathrm{m}$ platinum wire with a distance between the prongs of $\approx 0.5 \mathrm{~mm}$.

The probe was operated at $50 \%$ overheat and was calibrated in situ far outside the boundary layer against a Prandtl tube. The calibration function proposed by Johansson and Alfredsson, ${ }^{21}$ which compensates for the natural convection effects, was used. The calibration coefficients were determined from a best fit of the data to the calibration function.

A heat exchanger, positioned in the return circuit just after the fan, is capable to maintain a variation of the total pressure and the temperature below $\pm 0.06 \%$ and $\pm 0.05^{\circ} \mathrm{C}$, respectively. Therefore no temperature corrections for the anemometer signal were needed.

\section{B. Experimental setting}

The streaky boundary layer was generated by means of cylindrical roughness elements similar to those used and described in Ref. 17. To get a good spanwise homogeneity nine elements were periodically pasted on the plate surface. Relevant parameters in this study are the boundary layer scale $\delta$ corresponding to $\sqrt{x \nu / U_{\infty}}$, the height of the roughness element $k$, the cylinder diameter $d$, the spacing between the elements $\Delta z$, and the distance from the leading edge where the elements were located $x_{k}$ (see Fig. 1 with caption for an illustration of all parameters).

TS waves were generated by time periodic suction and blowing at the wall through a slot in a plug mounted in the plate. The slot, located at $x=190 \mathrm{~mm}$ from the leading edge, is $330 \mathrm{~mm}$ long in the spanwise direction and $0.8 \mathrm{~mm}$ wide.

The disturbance signal was generated by the computer through a $D / A$ board to an audio amplifier driving two loudspeakers, and the data set acquisitions were triggered by the reference signal from the waveform generator. The loudspeakers are connected to the disturbance source through 20 flexible tubes. A more thorough description of the disturbance generating system can be found in Ref. 22 .

The amplitude of the loudspeakers signal was manually adjusted for each frequency in order to keep the TS amplitudes below $0.7 \%$ of the $U_{\infty}$ at branch II. The spanwise uniformity of the generated waves was checked by measuring wall-normal distributions of TS amplitude at three different spanwise positions in the $2 \mathrm{D}$ case.

The structure of the flow was depicted by traversing the probe in the $y z$ plane, with a minimum number of points of $31 \times 18$, to $6-12$ different streamwise positions depending on 


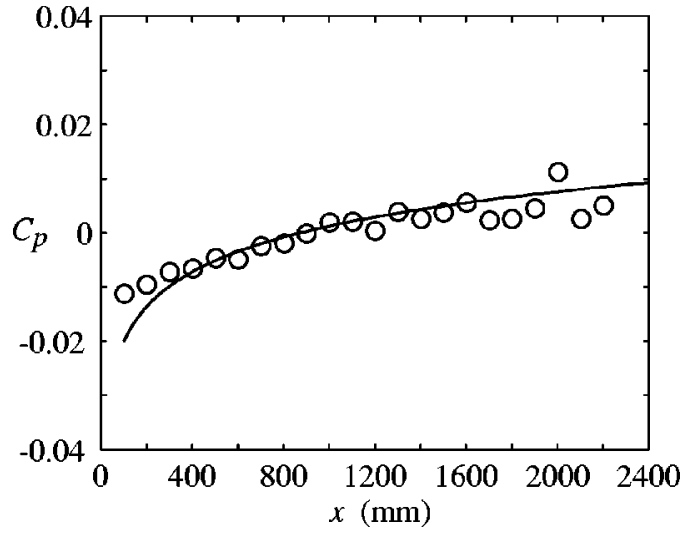

FIG. 2. External pressure gradient along the flat plate. Symbols are measured data and solid line is the solution to Eq. (2) with $m=-0.0046$ and $x_{0}=-1.3 \mathrm{~mm}$

the frequency. At each point the measure was obtained by acquiring three sets of samples with a frequency of $1 \mathrm{kHz}$.

\section{External pressure gradient}

The pressure gradient in the streamwise direction, generating the base flow, can be regulated by adjusting the ceiling of the test section. Care was devoted to find a zeropressure distribution and an iterative procedure allowed a small pressure gradient with a $C_{p}$ variation of about \pm 0.01 over a streamwise distance of $2.2 \mathrm{~m}$ (cf. Fig. 2). However, even though this value is very small and a Blasius-like base flow was generated, it was decided to take into account the small deviation by using a Falkner-Skan profile in the comparison with the theoretical stability analysis.

The Falkner-Skan (FS) family of self-similar mean velocity profiles is obtained by varying the external pressure gradient and assuming a free stream $\left(U_{\infty}\right)$ variation according to

$$
U_{\infty}(x) \sim\left(x-x_{0}\right)^{m},
$$

where $m$ is the imposed pressure gradient ( $m>0$ corresponds to a favorable gradient and $m<0$ to an adverse). A virtual origin is accounted for through $x_{0}$, which is determined by comparing the measured displacement thickness $\delta^{*}$ with the theoretical value for the specific pressure gradient under consideration. Since the shape factor $H$ is uniquely defined by $m$, its value is here determined by choosing the FS profile that corresponds to the value of $H=2.61$ calculated from the experimental data at $\operatorname{Re}=601$. This is the $\operatorname{Re}$ from where the region of self-similar velocity profiles seems to have started (cf. Fig. 4). This procedure gives a value of $m=-0.0046$.
Now, a comparison of the theoretical and the experimental $\delta^{*}$ gives $x_{0}=-1.3 \mathrm{~mm}$ for $U_{\infty}=5 \mathrm{~ms}^{-1}$, which is used to correct the data throughout this paper.

The pressure coefficient $C_{p}$ is connected to $m$ through the differential equation

$$
\frac{\partial C_{p}}{\partial x}+2 \frac{m}{\left(x-x_{0}\right)}\left(1-C_{p}\right)=0
$$

which is derived from potential flow theory together with Eq. (1). In Fig. 2 the choice of $m$ is verified by plotting the $C_{p}$ values (symbols), calculated from the velocity measurements, together with the solution of the differential Eq. (2) (solid line), where the initial condition has been chosen to fit the experimental data. Note that $m$ can also be determined directly with a least square curve fit based on Eq. (1), which would result in $m=-0.0031$. This value, however, would give a worse agreement in the stability analysis comparison considered in Sec. IV.

\section{CHARACTERISTICS OF THE BASE FLOWS INVESTIGATED}

This section reports the three-laminar base flows whose stability have been investigated. The reference case is a FS type of boundary layer with a "weak" adverse pressure gradient, as discussed above, and will from here on be denoted case $R$. Two "similar" (to each other) streaky base flows consisting of alternating high and low speed streaks subject to the same pressure gradient as the $R$ case are also considered. These streaky flows are generated by cylindrical roughness elements of different heights, namely, $k=1.3$ and $1.4 \mathrm{~mm}$, pasted on the plate with a spanwise spacing $\Delta z$ $=14.7 \mathrm{~mm}$. The two roughness heights correspond to $k / \delta_{k}$ equal to 2.65 and 2.86 , where $\delta_{k}$ is the boundary layer scale at the position of the roughness element array $x_{k}$, and will be denoted cases $S_{1}$ and $S_{2}$, respectively. The free-stream velocity was kept constant at $5 \mathrm{~ms}^{-1}$ in all the experiments, resulting in maximum streak amplitudes of $11.6 \%$ and $12.4 \%$ of $U_{\infty}$. All the characteristics of the three base flows $R, S_{1}$, and $S_{2}$ reported in this section are summarized in Table I.

\section{A. The effect of the roughness elements on the base flow}

An important parameter related to the maximum achievable streak amplitude is the roughness height Reynolds number $\operatorname{Re}_{k}=U_{(y=k)} k / \nu$ (see, e.g., the work by White and $\left.\operatorname{Ergin}^{23}\right)$. There are two different ways of increasing $\operatorname{Re}_{k}$, either by increasing $U_{\infty}$ or by increasing $k$. On the contrary to the previous experiment, ${ }^{17}$ here $U_{\infty}$ is kept constant and $k$ has

TABLE I. Summary of the experimental base flow parameters. $A_{\mathrm{ST}}^{*}$ is the maximum streak amplitude in percentage of $U_{\infty}$. $\delta_{k}$ is evaluated at the position of the roughness elements $x=x_{k}$.

\begin{tabular}{ccccrrrc}
\hline \hline Case & $U_{\infty}\left(\mathrm{ms}^{-1}\right)$ & $k(\mathrm{~mm})$ & $k / \delta_{k}$ & $\operatorname{Re}_{k}$ & $\operatorname{Re}$ & $\beta$ & $A_{\mathrm{ST}}^{*}(\%)$ \\
\hline$R$ & 5 & 0 & 0 & 0 & 0 & 0 & 0 \\
$S_{1}$ & 5 & 1.3 & 2.65 & 340 & 163 & 0.21 & 11.6 \\
$S_{2}$ & 5 & 1.4 & 2.86 & 385 & 163 & 0.21 & 12.4 \\
\hline \hline
\end{tabular}



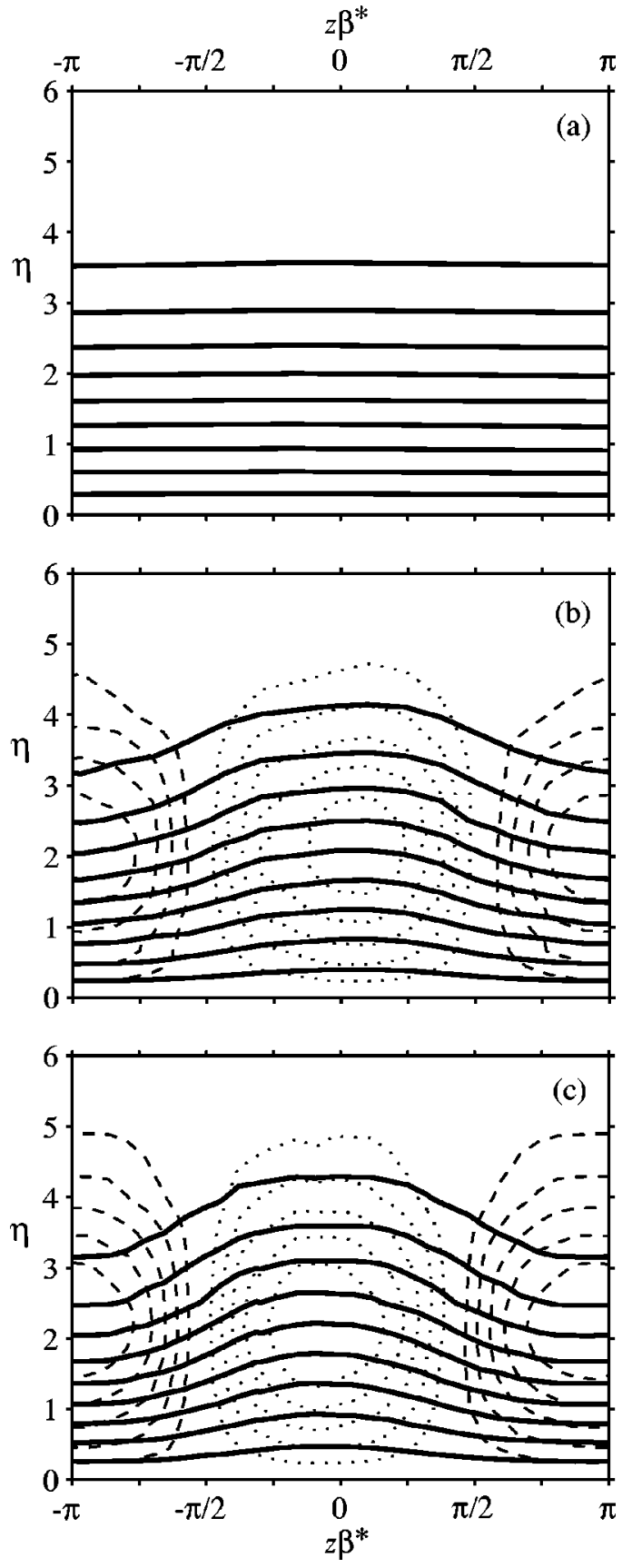

FIG. 3. Base flows (at maximum streak amplitude) for the $R$ (a) and the two studied streaky flows $S_{1}$ and $S_{2}$ (b, c) of increasing roughness height. Solid bold lines are velocity contours with an increament of $U / U_{\infty}=0.1$ from the wall. Dotted and dashed lines are negative and positive contours of spanwise mean deviation $\left(U-\bar{U}_{z}\right) / U_{\infty}= \pm\left[\begin{array}{lllll}0.02 & 0.04 & 0.06 & 0.08 & 0.1\end{array}\right] . \beta^{*}=0.43 \mathrm{~mm}^{-1}$.

been changed. This is in order to keep the same physical boundary layer thickness and the same location of branches I and II in the stability analysis for a given frequency, which should make a direct comparison completely fair. For the TS stability analysis we use the Reynolds number based on the boundary layer scale, namely, $\operatorname{Re}=U_{\infty} \delta / \nu$.

The spanwise array of cylindrical roughness elements modulates the 2D base flow into a 3D flow. In Fig. 3 we display $y z$ scans (i.e., cross-stream planes) of both the mean streamwise velocity (solid bold lines) and of the deviation from the spanwise mean (dotted and dashed lines) for the
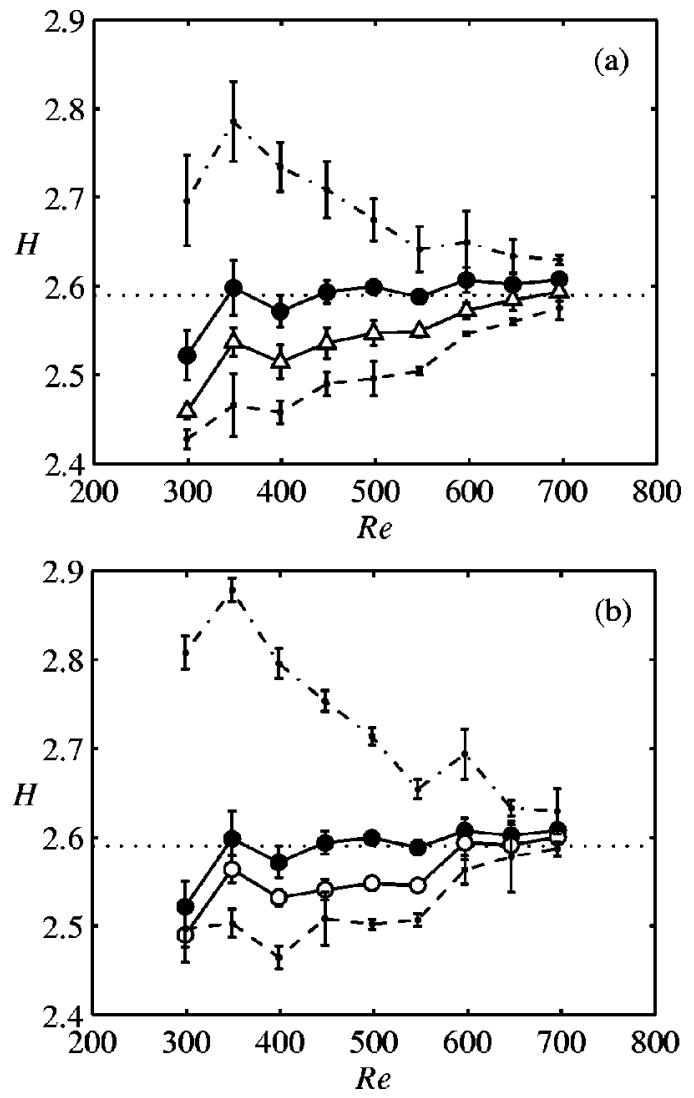

FIG. 4. (a) Spanwise averaged shape factors for the $R$ and $S_{1}$ cases with $(\mathbf{O})$ and $(\triangle)$ symbols, respectively. (b) Spanwise averaged shape factors for the $R$ and $S_{2}$ cases with (O) and (O) symbols, respectively. Dotted, dash-dotted, and dashed lines correspond to the theoretical Blasius and the experimental low- and high-speed streaks, respectively. The error bars indicate the standard deviation calculated from three sets of data.

three configurations considered. The streamwise locations correspond to the streamwise position of maximum streak amplitude, i.e., at $\approx \operatorname{Re}=401$. Note that the undisturbed boundary layer flow in Fig. 3(a) also was scanned at its streamwise station.

In Figs. 4(a) and 4(b) the streamwise evolution of the spanwise averaged shape factor $H$ is shown for the cases $S_{1}$ and $S_{2}$, together with the $R$ case plotted with $(\bullet)$ symbols. The shape factors in the low- and high-speed streaks are also reported in the plots by using dash-dotted and dashed lines, respectively, while the dotted line corresponds to the theoretical Blasius. Note, here, how close the $R$ case is to a Blasius boundary layer. It is clearly seen that the spanwise averaged shape factor is decreased when the streaks are present, implying a fuller and thereby a more stable wall-normal mean velocity profile from a 2D point of view. However, as shown in Ref. 8, such an analysis alone is not able to explain the TS stabilization observed in the presence of streamwise streaks, which is infact induced by the work of the spanwise shear associated with the streak profile. Moreover, there is no noticeable difference in the spanwise averaged $H$ between the two streaky flows. 


\section{B. Suboptimal streaks}

The nonmodal growth of steady and spanwise periodic streamwise streaks in a flat plate boundary layer has previously been studied and the discrepancy between the experimental streaks and the theoretical optimally growing streaks was clarified (see the work by Fransson et al. ${ }^{17}$ ). The key factor was found to be the wall-normal location and the extension of the laminar standing streamwise vortices inducing the streaks in an experimentally feasible configuration. The theoretical approach followed in Ref. 17, here denoted suboptimal perturbation theory, considers the evolution of the optimal upstream vortices calculated at the same downstream position $\left(X_{0}\right)$ as the roughness elements but compressed or stretched in the wall-normal direction through a parameter $c$ (stretched $1<c<1$ compressed). In this way, it was possible to reproduce the features of the experimentally generated streaks and conclude that the difference with the optimal perturbation is due to the fact that the upstream vortices are located closer to the wall. In that paper it was also shown that, even for the high amplitude streaks considered, after a complex development in the region close to the elements, the spanwise fundamental mode of the streak profile was clearly the dominating mode downstream of the amplitude maximum. Therefore the following streak amplitude $A_{\mathrm{ST}}$ definition appears justified.

$$
A_{\mathrm{ST}}=\max _{y}\left\{\left(U(y)_{\text {high }}-U(y)_{\text {low }}\right) / 2\right\} / U_{\infty},
$$

where $U(y)_{\text {high }}$ and $U(y)_{\text {low }}$ denote the velocity profiles in the high and low speed regions, respectively.

The streamwise evolution of the streak amplitude $A_{\mathrm{ST}}$ is plotted in Fig. 5 versus both $\operatorname{Re}$ and $X$. The rescaling to the nondimensional $x$ coordinate $X=\left(\beta^{*} \delta / \beta_{\text {opt }}\right)^{2}$ has experimentally been shown to produce a universal streak behavior. ${ }^{5,17}$ The validity of the scaling above, derived directly from the boundary layer approximation, implies that the streaks do obey the boundary layer equations except in a small region near the roughness elements. $\beta_{\text {opt }}$ is simply a proportionality/ scaling constant, which can be chosen freely, usually selected such that at $X=1$ the spanwise wavenumber is the one that produces maximum energy growth according to optimal perturbation theory (i.e., $\beta_{\text {opt }}$ equals 0.45 ). For a thorough account on this scaling the interested reader is referred to Ref. 17. In Fig. 5(a) it is seen that the only difference between the two cases $S_{1}$ and $S_{2}$ is the streak strength, since the two curves essentially are parallel. Normalized to unity and plotted versus $X$ the two streak amplitude curves fall on top of each other, confirming also in this case the validity of the boundary layer approximation. Furthermore, the amplitude evolution is in good agreement with the amplitude curve predicted by suboptimal perturbation theory with a stretching parameter of $c=0.75$. The difference at the first streamwise measurement station is in fact due to the complicated flow behavior observed just downstream of the roughness elements. In Fig. 5(c) the wall-normal position of the maximum streak amplitude is plotted versus the downstream distance to show that the streaks are not self-similar as also predicted by the theory (solid line). The departure from self-similarity is, however, small as illustrated in Fig. 6 where the entire wall-
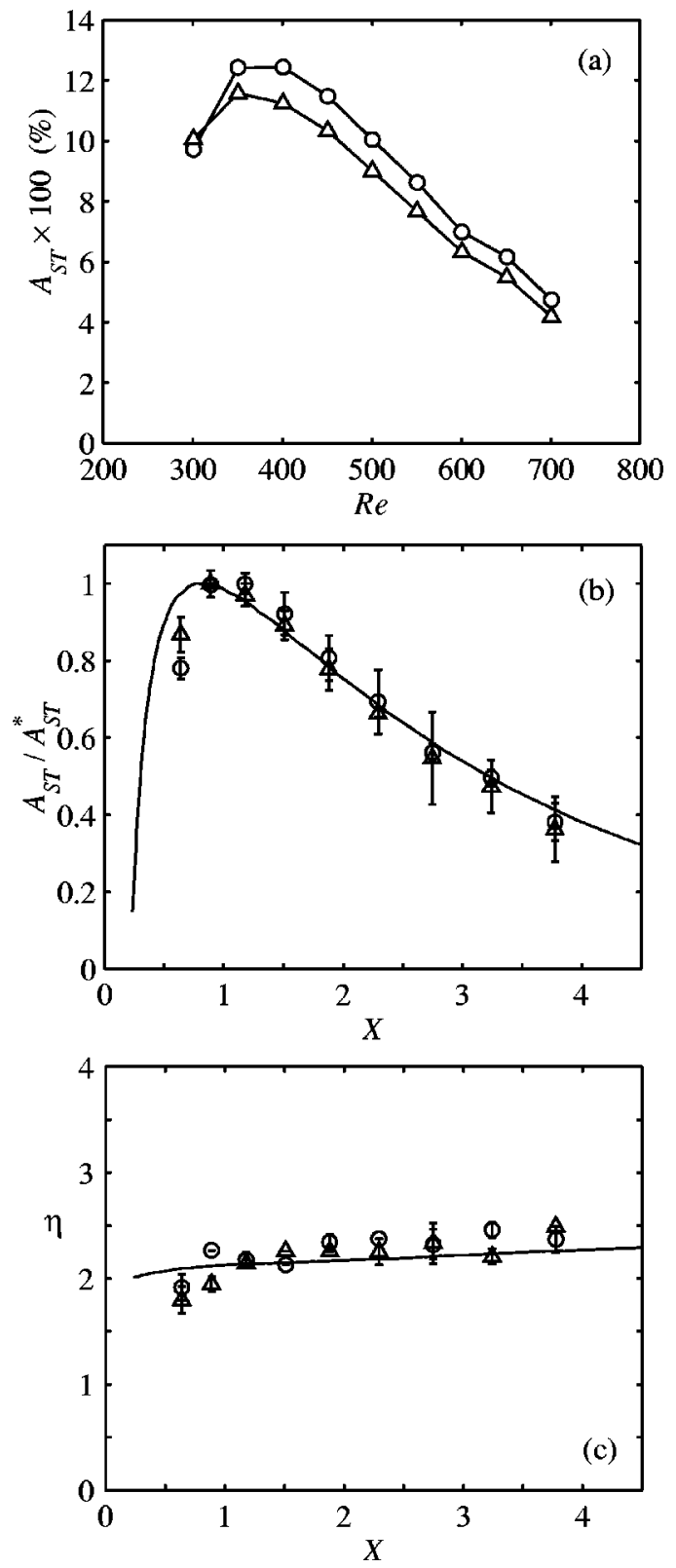

FIG. 5. (a) Streak amplitude $\left(A_{\mathrm{ST}}\right)$ vs Re. (b) $A_{\mathrm{ST}}$ scaled with its maximum and plotted vs $X$. (c) Position of maximum $A_{\mathrm{ST}}$ above the wall. The symbols $(\triangle, \bigcirc)$ correspond to the $S_{1}$ and $S_{2}$ cases, respectively. Solid lines in (b) and (c) correspond to suboptimal perturbation theory with $c=0.75$ and $X_{0}$ $=0.218$ (see text for reference). The error bars indicate the standard deviation calculated from three sets of data.

normal perturbation profiles are plotted for the different streamwise stations shown in Fig. 5 and compared with the theoretical profile at $X=2$. Note that the experimental perturbation profiles are the spatial root mean square profiles in the spanwise direction (and not in time).

\section{The stability of the generated streaks}

The stability of the 3D streaky base flow was checked in order to be sure that the TS waves are generated under steady and stable conditions, and not influenced by nonlinear effects caused by preexisting secondary instabilities on the streaks nor by shedding from the roughness elements.

For high enough $\operatorname{Re}_{k}$ a shedding on the roughness ele- 


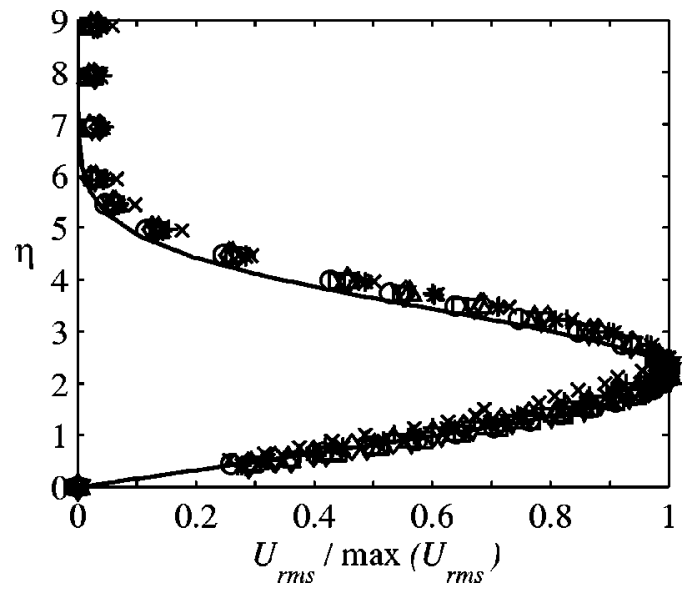

FIG. 6. Wall-normal perturbation profiles. Symbols correspond to different $X$ positions of the $S_{2}$ case and the solid line corresponds to the suboptimal theoretical profile at $X=2$.

ments sets in. Whether this is a hairpin vortex type over the top edge as discussed by Acarlar and Smith ${ }^{24}$ or if it is a von Kármán type of vortex shedding cannot be concluded from the type of measurements performed here. The shedding frequency is around $11 \mathrm{~Hz}$ and gives a clear peak in the energy spectrum. Here, we can only conclude that the limitation to create steady and stable streaks of higher amplitudes is not due to an inviscid instability of the streaks themselves, but rather due to an instability associated to the generating device (i.e., the roughness elements).

The intermittency function $\gamma$, computed as described in the work by Fransson et al. ${ }^{9}$ is determined for increasing $\mathrm{Re}_{k}$ in order to show a local measure of the flow instability. For the collection of those data the hot-wire probe was positioned at a fixed downstream location $(x=326 \mathrm{~mm})$, behind the middle roughness element and close to the wall, while varying the free-stream velocity. The exact position above the wall is not crucial for the repeatability since the intermittency is fairly constant up to at least $y / \delta^{*}=2$ (cf. the work by Matsubara et $a l .{ }^{25}$ ). The free-stream velocity was varied between 4.8 and $5.8 \mathrm{~ms}^{-1}$ (resulting in $368<\mathrm{Re}_{k}<468$ ) and at each velocity 40000 samples were collected at a rate of $1000 \mathrm{~Hz}$, which were used for the intermittency calculations.

In Fig. 7 the intermittency behind the middle roughness element, i.e., in a high-speed streak, is plotted versus $\operatorname{Re}_{k}$ and Re. From this figure the critical $\operatorname{Re}_{k}$ (defined as where the intermittency equals 0.5 ) can be determined to be 422 for this particular roughness element array in the MTL wind tunnel. Note that the largest $\operatorname{Re}_{k}$ that later was used for the TS wave experiments was 385 , i.e., well below the critical value.

\section{3D MODULATED TOLLMIEN-SCHLICHTING WAVES}

In order to illustrate the effect of streaks on TS waves five different frequencies were investigated for both the $R$ and the $S_{2}$ cases, while the $S_{1}$ case only was investigated at $F=130$. Throughout this paper the nondimensionalized frequency $F=\left(\omega \nu / U_{\infty}^{2}\right) \times 10^{6}$ will be used. In Fig. 8 the neutral

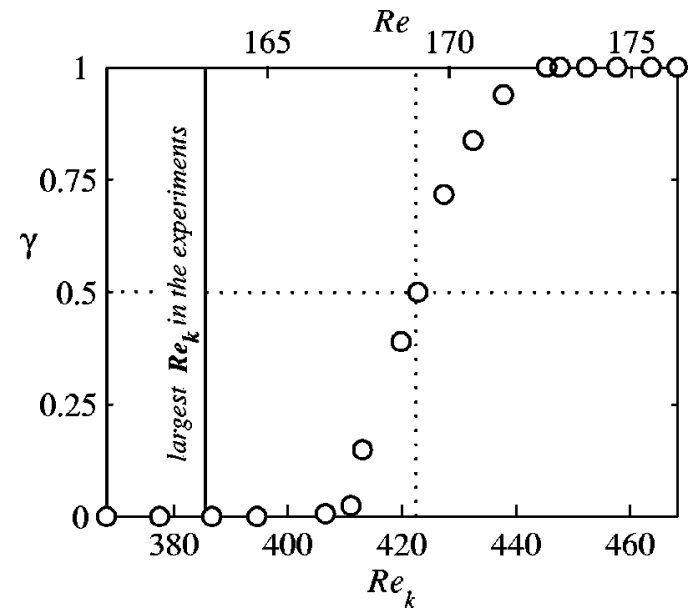

FIG. 7. Intermittency factor $v s \mathrm{Re}_{k}$, which has been varied by changing $U_{\infty}$ in the interval $4.8-5.8 \mathrm{~ms}^{-1}$.

stability curves for the FS and the Blasius boundary layers are shown together with five horizontal solid lines, which indicate the five studied frequencies.

\section{A. Shape of the eigenfunctions}

The 2D generated TS waves are redistributed in the spanwise direction at the instant they enter the boundary layer in order to comply with the 3D modulated base flow. An example of the fundamental TS mode $(F=110)$ distribution close to branch II is illustrated in Fig. 9, where the dotted lines indicate the location of low- and high-speed streaks (see caption for contour levels). Here, the data in the figure has been smoothed by local spatial averaging. In contrast to the single peak profile in the high-speed region, which resembles the amplitude profile in a $2 \mathrm{D}$ base flow, a two-peak profile is observed in the low speed region. This is in agreement with previous studies ${ }^{15,16}$ and is best illustrated in Fig. 10(a) where the wall-normal profiles are plotted at three spanwise locations together with the spanwise averaged profile (bold solid line). The three spanwise stations considered correspond to the high- and low-speed streaks and to the position of maximum spanwise shear, denoted "shear speed."

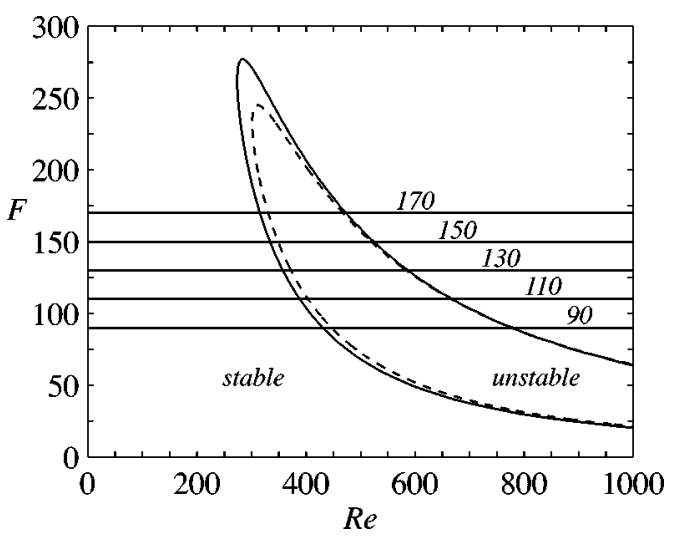

FIG. 8. Neutral stability curves for the FS (solid line), with $m=-0.0046$, and the Blasius boundary layers (dashed line). The horizontal lines indicate the five frequencies studied in the present investigation. 


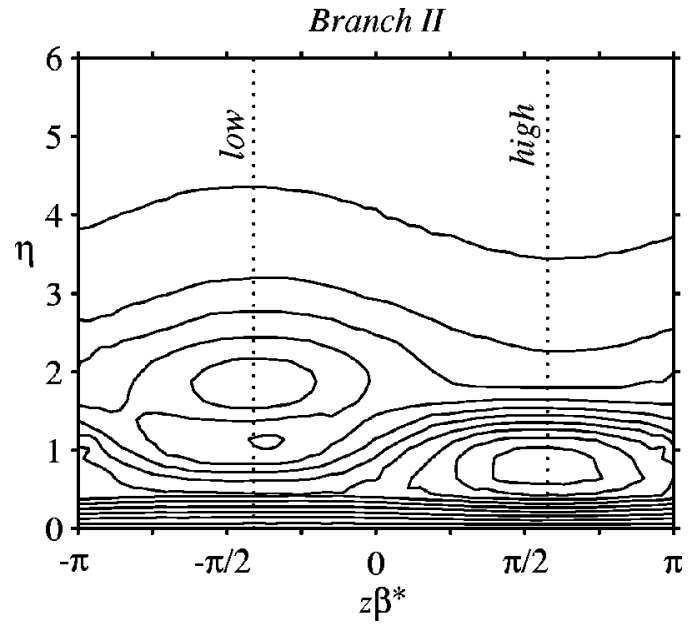

FIG. 9. Experimental cross sectional measure of the streaky TS amplitude for $F=110$ and close to branch II $(\operatorname{Re}=651)$. Dotted lines indicate the location of low- and high-speed streaks. The contour levels are $(0.5,1,1.5,2,2.5,3,3.5,4,4.5) \times 10^{-3}$ and $\beta^{*}=0.46 \mathrm{~mm}^{-1}$.

In Fig. 10(b) the corresponding wall-normal phase distributions are shown and it is clear that the distribution is much more complicated below $\eta=3$ as compared to the $2 \mathrm{D}$ case.

The streamwise evolution of the perturbation is shown in Fig. 11 both at the high- (a) and at the low- (b) speed locations. In the high-speed region the variation is not so dramatic as compared to the low-speed region where it is seen that the amplitude of the outer peak decreases by a factor of 2 relative to the inner peak. It can also be seen that approaching branch II the shape variations diminish and that the maximum in the high-speed region is located at $\eta=0.8$ inde-
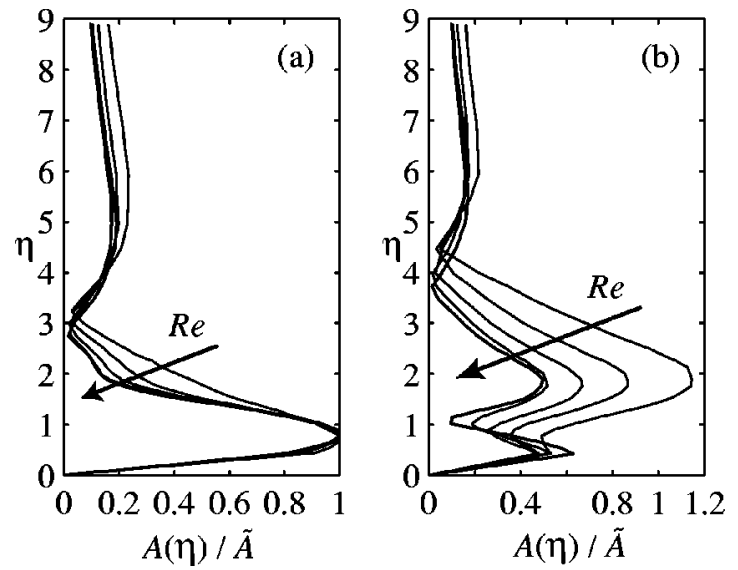

FIG. 11. Wall-normal amplitude profile evolution for $F=110$. (a) High- and (b) low-speed location. $\operatorname{Re}=451,501,551,601,651 . \tilde{A}$ corresponds to the inner peak value in the high-speed location for each Re.

pendent of Re. Note here that the outer peak may reach higher amplitudes in the low-speed region than in the highspeed region around branch I, this result is, however, not general but was also observed for $F=90$, i.e., for the two lowest frequencies.

The Fourier content of the 3D modulated TS waves is also analyzed. The wall-normal profile of the spanwise mean and of the first two spanwise harmonics are shown in Fig. 12 at the two streamwise stations close to branch I and branch II, and for the five frequencies under consideration. The bold solid lines in the figures correspond to the averaged profile of all the experimental data at each streamwise location. At branch I a typical M-shape structure is clearly visible in the
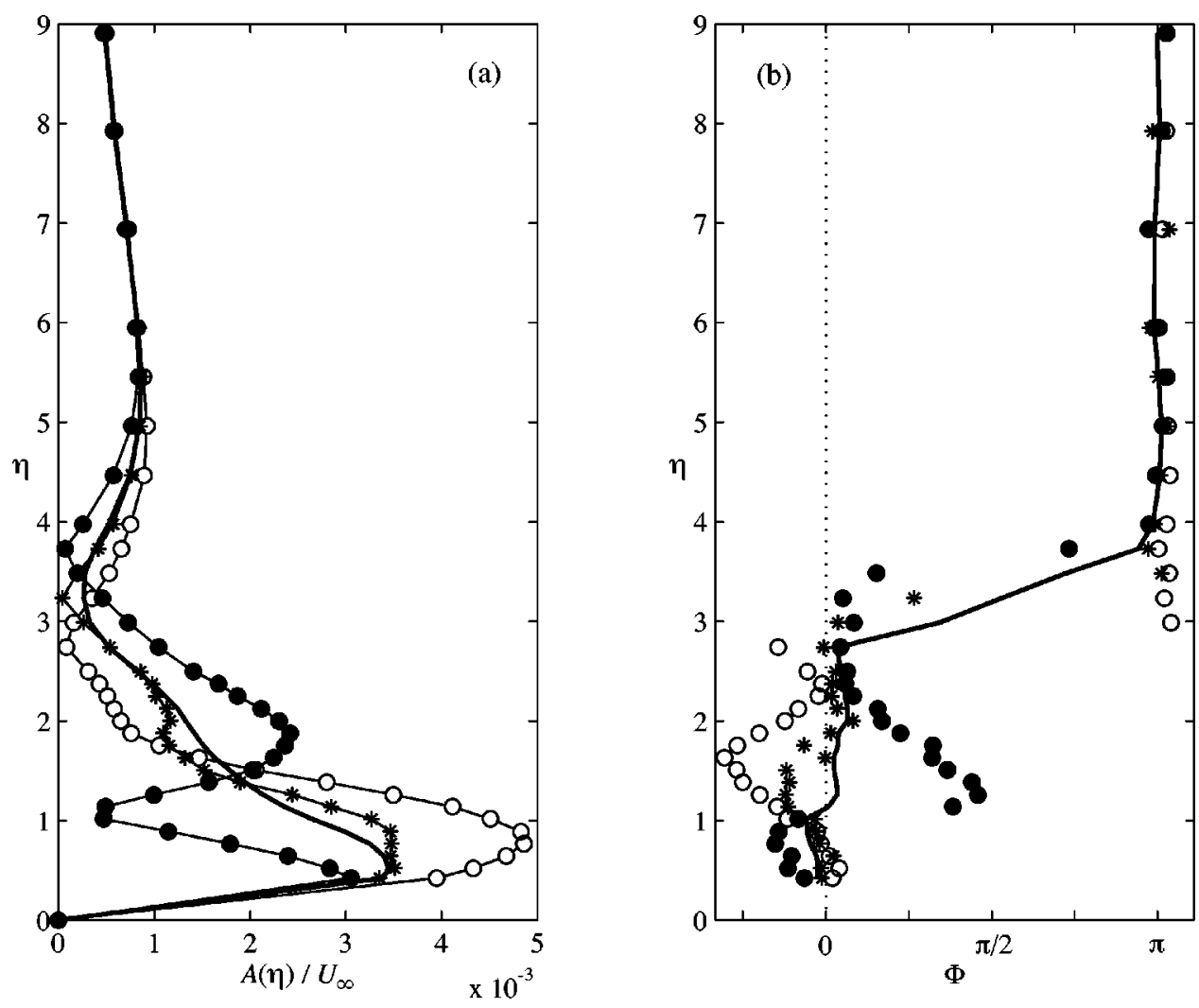

FIG. 10. (a) Wall-normal TS amplitude profiles and (b) corresponding phase distributions of Fig. 9. (○), (๑), and (*) correspond to the high-, the low-, and the shear-speed spanwise locations. Bold solid line correspond to the spanwise averaged profile. $F=110$ and $\operatorname{Re}=651$. 

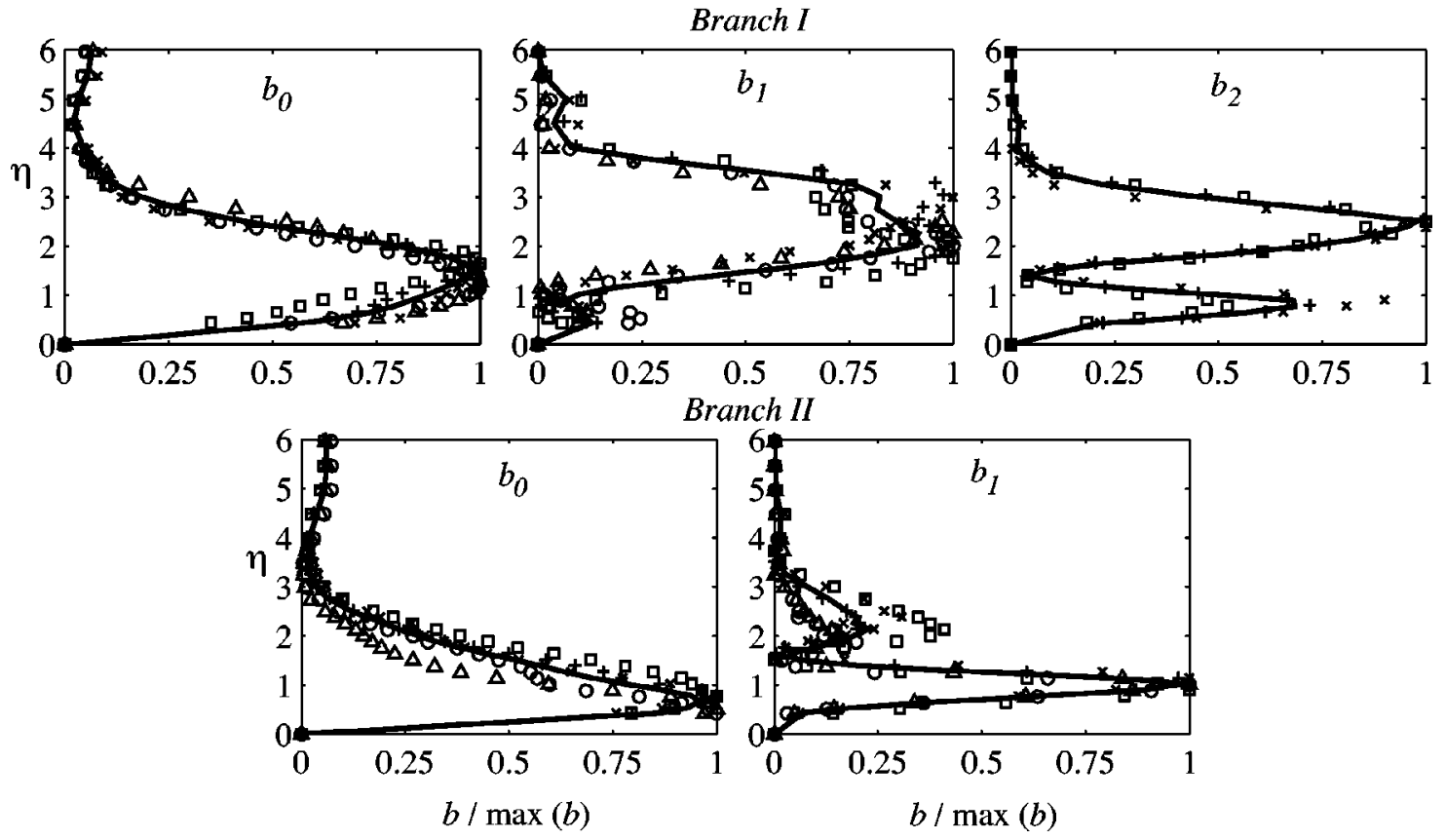

FIG. 12. Fundamental mode decomposition at branches I and II. $(\square) F=130,(\triangle) F=110,(+) F=150,(\times) F=170,(\bigcirc) F=90$. Bold solid lines correspond to the averaged profile of the experimental data.

first $\left(b_{1}\right)$ and second harmonic $\left(b_{2}\right)$, being more apparent in the latter. Further downstream the $b_{2}$ component becomes negligible and the profile scattered, and therefore it is not reported in the figure, while the outer peak of $b_{1}$ is still apparent even if of smaller relative amplitude.

The downstream evolution of the energy associated to each spanwise mode, defined as

$$
B_{j}=\int_{0}^{\eta^{*}} b_{j} d \eta
$$

is shown in Fig. 13. Here $\eta^{*}=9$ is used throughout this paper if otherwise is not stated. In Fig. 13(a) it can be seen that most of the energy is in the spanwise independent mode. In Fig. 13(b) the same data are divided by the energy $B_{j}$ at branch I $\left(B_{j, 0}\right)$ in order to follow the development of the relative amplitude. It can be seen that the first harmonic grows faster at the beginning while it decays faster close to branch II. This may be related to the corresponding streak evolution. As the streak amplitude increases, the TS becomes more $3 \mathrm{D}$ and we have a relative increase of $B_{1}$. On the contrary, further downstream with lower streak amplitudes, the flow is less modulated and the TS waves also tend to return to their original $2 \mathrm{D}$ structure. The same behavior is observed for the other frequencies as well.

\section{B. Amplitude measure and its downstream evolution}

Several different TS amplitude/energy measures can be found in the literature, and in the work by Fasel and Konzelmann ${ }^{26}$ a thorough investigation of the different measures has been performed. Experimentally one usually considers a rather simple measure, namely, the local maximum amplitude or energy of the streamwise velocity component at fixed spanwise locations since both the wall-normal and the
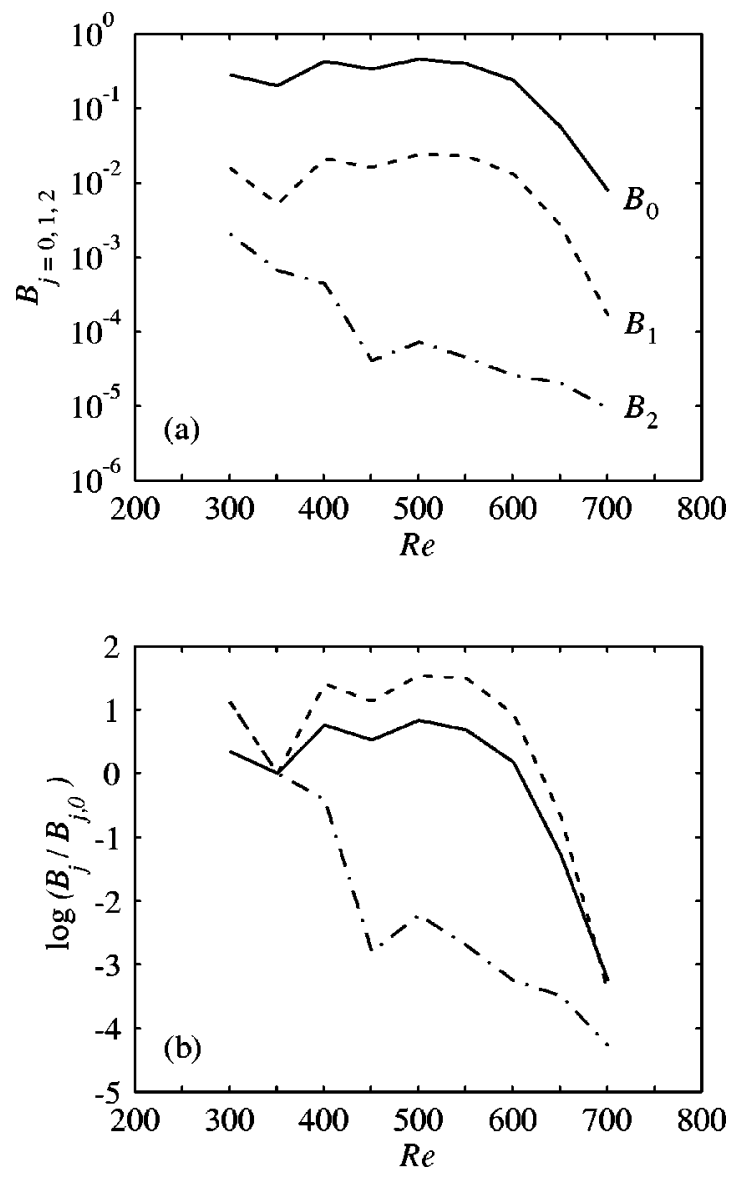

FIG. 13. Integrated amplitude measure of the fundamental mode harmonics $b_{j=0,1,2}$. (a) shows the absolute value and (b) the relative growth of the different harmonics. The different lines correspond to $B_{j=0,1,2}$ and are shown in (a). $F=130$. 


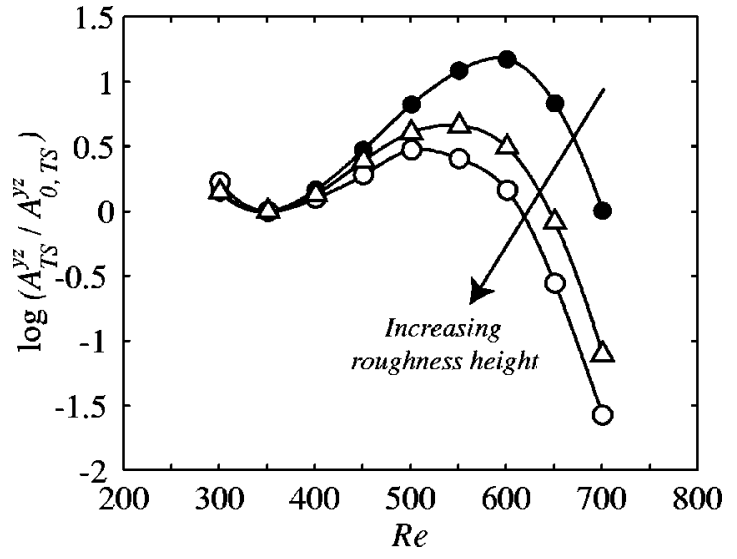

FIG. 14. TS amplification curves for the $R$ case $(\bullet)$ and for successively increasing roughness heights $S_{1}$ and $S_{2}(\triangle, \bigcirc) . F=130$. Solid lines are fitted spline curves to the experimental data.

spanwise perturbation components are difficult to measure. However in a 3D periodic base flow, as studied here, the wave eigenfunction depends on both the wall-normal and spanwise directions, being periodic in the latter, and therefore it is necessary to integrate the amplitude in the crossstream plane to obtain a proper measure which also can be used for comparisons with waves arising in 2D base flows. Here we will use the following expression as a measure of the modulated TS wave amplitude:

$$
A_{\mathrm{TS}}^{y z}=\int_{0}^{2 \pi} \int_{0}^{\eta^{*}} \frac{A_{\mathrm{TS}}\left(\eta, \beta_{z}\right)}{U_{\infty}} d \eta d \beta_{z} .
$$

Note that other amplitude measures, as discussed in the Introduction, may give rise to different results. However, to clarify these differences a separate section has been devoted to this problem (cf. Sec. IV C).

The present experimental data with the cross sectional integral amplitude measure $\left(A_{\mathrm{TS}}^{y z}\right)$ defined above normalized with ditto measure at branch $\left(A_{0, \mathrm{TS}}^{y z}\right)$ shows unambiguously that the TS wave growth is damped when these waves evolve in a 3D sinusoidal base flow. Furthermore, Fig. 14 shows the first experimental result ever on successively increased damping of the TS waves with increasing streak amplitude. Recall, that the only difference between the $S_{1}$ and $S_{2}$ cases is the roughness element height, all other parameters are kept constant. Figure 15 reveals that the damping is observed for all frequencies studied. For low frequencies the effect seems to be smaller which can be explained by the relatively low streak amplitudes $\left(A_{\mathrm{ST}}<4 \%\right.$ of $\left.U_{\infty}\right)$ attained around branch II in those cases. Note that in the present study the maximum streak amplitude occurs close to branch I while in the theoretical works by Cossu and Brandt ${ }^{7,8}$ the maximum streak amplitude is close to branch II. Together with the higher streak amplitudes considered in Refs. 7 and 8, this may explain the stronger stabilisation observed in the latter works, where TS waves are even completely suppressed.

It can be seen that the steady streaks induced by the roughness elements slightly delay the onset of the viscous TS instability (branch I), and that the streamwise distance of downstream growth (branch II) is decreased. The stabilizing
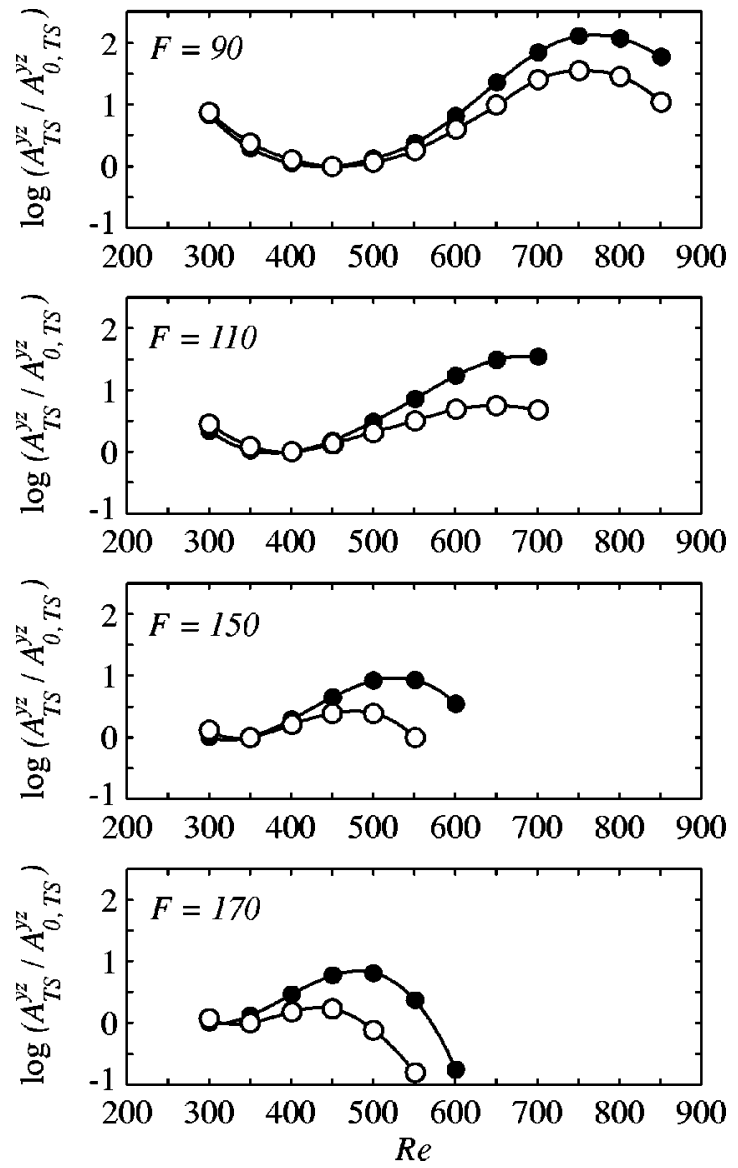

FIG. 15. TS amplification curves for the $R$ case $(0)$ and for the $S_{2}$ case $(\bigcirc)$ for different frequencies. Solid lines are fitted spline curves to the experimental data.

effect is not only seen by the different location of the neutral points but it can also be correctly, and maybe most significantly, quantified by considering the total amplification of the instability waves.

The two consequent measurement points in Figs. 14 and 15 giving maximum growth, for each frequency, are used for estimating the overall growth factor $\left(\alpha_{i}=\alpha_{i}^{*} \delta\right)$. Note that this procedure, considering two discrete measurement points to estimate the maximum slope, would ideally always result in a growth smaller than the true one. In Fig. 16 the growth factors in the 3D modulated base flows are plotted versus the frequency and compared with the $R$ case. The dashed line corresponds to the FS growth factor curve from linear parallel theory (cf. Sec. IV E) and the experimental measure has been calculated as

$$
-\alpha_{i}=\frac{1}{2} \frac{d\left\{\log \left(A_{\mathrm{TS}}^{y z} / A_{0, \mathrm{TS}}^{y z}\right)\right\}}{d \operatorname{Re}} .
$$

To end this section we investigate the effect of the wallnormal truncation value $\eta^{*}$ in the cross-stream amplitude measure [Eq. (4)]. In Fig. 17 the relative change of $A_{\mathrm{TS}}^{y z}$ between the $R$ case and the $S_{2}$ case for $F=130$ at branch II is shown versus $\eta^{*}$. One may conclude that the choice of $\eta^{*}$ has a small effect on the value of $A_{\mathrm{TS}}^{y z} / A_{0, \mathrm{TS}}^{y z}$. If $\eta^{*}$ is chosen 


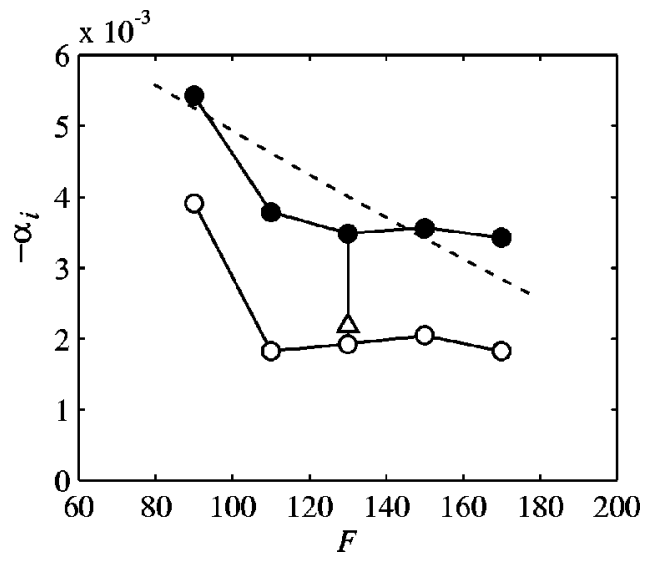

FIG. 16. Experimental TS growth factors $\left(\alpha_{i}^{*} \delta\right)$ vs frequency. $(\bullet, \triangle, \bigcirc)$ correspond to the $\left(R, S_{1}, S_{2}\right)$ cases, respectively. Dashed line is the theoretical FS corresponding to the $R$ case.

to be 6 instead of 9, as an example, the $R$ symbol in Fig. 14 would approach the $S_{2}$ symbol by $\approx 1.5 \%$ of the relative distance at branch II.

\section{Effect of the amplitude measure definition}

Previous investigators of TS waves in streaky base flows have not considered the entire cross-stream plane when calculating the amplitude as done in this investigation. As mentioned in the Introduction and emphasized here, either the $z$ or the $y$ section has been kept constant (cf., e.g., the work by Kachanov and Tararykin ${ }^{15}$ and Bakchinov et al., ${ }^{16}$ respectively). In this section we intend to elucidate the differences between the different amplitude definitions by reporting the maximum, the $y$-integral, and the $z$-integral, measures.

The maximum is the traditionally used measure in $2 \mathrm{D}$ base flows and has been shown to be the measure that agrees best with spatial linear parallel stability theory (LPT) when comparing amplification curves (see Sec. IV E for a thorough comparison between experiments and LPT). This measure is simply the maximum value of the inner peak at a fixed $z$ position and will be denoted $A_{\mathrm{TS}}^{\max }$. Note here that the

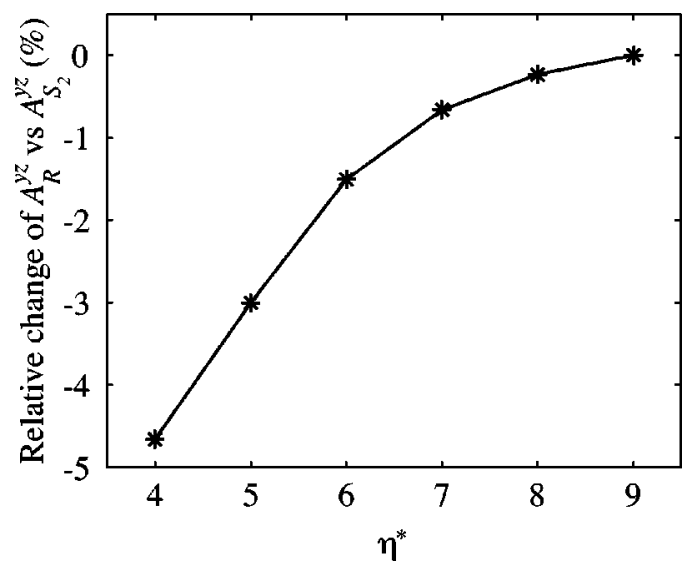

FIG. 17. Effect of wall-normal truncation in calculating the amplitude measure Eq. (4). Confer the text for an example about how to interpret this figure. TS cross-stream planes considered are for $F=130$ at branch II.
3D-TS amplitude profile in the low speed is $\mathrm{M}$ shaped, i.e., it has an inner and an outer peak apart from the upper classical peak at $\eta \sim 5-6$.

As the $y$-integral measure we consider the integral of the wall-normal TS profiles in the high- and low-speed streaks and in the shear region between these two. The $z$-integral measure considers the integral over one period of the spanwise TS profiles at fixed $\eta(=0.8)$. This value of $\eta$ corresponds to the inner peak value in the high-speed region, which is seen to be independent of Re. Note that Kachanov and Tararykin ${ }^{15}$ used $\eta=0.9$, which corresponded to the location of maximum TS amplitude in the high-speed streak in their particular streaky base flow. The $y$ - and $z$-integral measures will be denoted $A_{\mathrm{TS}}^{y}$ and $A_{\mathrm{TS}}^{z}$, respectively.

In Fig. 18 all three amplitude measures just mentioned are shown for the perturbations with $F=130$. The maximum measure shown in Fig. 18(a) reveals that the strongest growth is found in the high-speed region (+ symbols), which is also the area where the highest absolute amplitude around branch II is found. The maximum amplitude at the shear location (* symbols) is in between the values obtained considering the outer ( $X$ and dash-dotted line) and the inner peak $(X$ and solid line) of the perturbation in the low-speed streak. Note that branch II is moved upstreams for all the local spanwise measures when compared to the $R$ case ( symbols) and that the relative growth also is longest for ditto. Figure 18(b) shows that the $y$-integral amplitude measure experiences a smaller growth than the maximum measure (the reason is discussed in Sec. IV E), and that the strongest growth is found in the high-speed region, followed by the shear, and then the low-speed region. The $z$-integral amplitude measure also indicates that there is a damping effect and is illustrated in Fig. 18(c). Note also that in contrast to the eigenfunction in the streaky base flow the inner maximum of a classical 2D-TS wave moves towards the wall with increasing Re. For $F=130$ this movement is from about $\eta=1.5$ at branch I to around $\eta=0.5$ at branch II. Therefore the maximum of the inner peak has also been followed and used as the wall-normal position where the $z$ integration is performed. The corresponding relative amplification curve for the $R$ case is depicted by the dashed line in Fig. 8(c). Interestingly this curve is slightly shifted downstream when compared to the (O)-symbol case where $\eta$ has been kept constant. The observations above are valid for the other frequencies considered as well and may be considered as a general result for this particular 3D base flow $\left(S_{2}\right)$.

As mentioned briefly above the difference between the different amplitude measures come from the fact that the shape of the mode changes under the combined effect of Re (viscosity effect) and of the local streak profile ( $X$ effect).

\section{Phase speed}

The phase speeds of the 3D-TS waves are computed by considering the velocity at the three spanwise stations corresponding to the low- and high-speed positions and to the location of maximum spanwise shear. Measurements are taken both close to the wall and further away in the boundary layer, in both cases at constant $\eta$. As shown in Fig. 10(b), the 

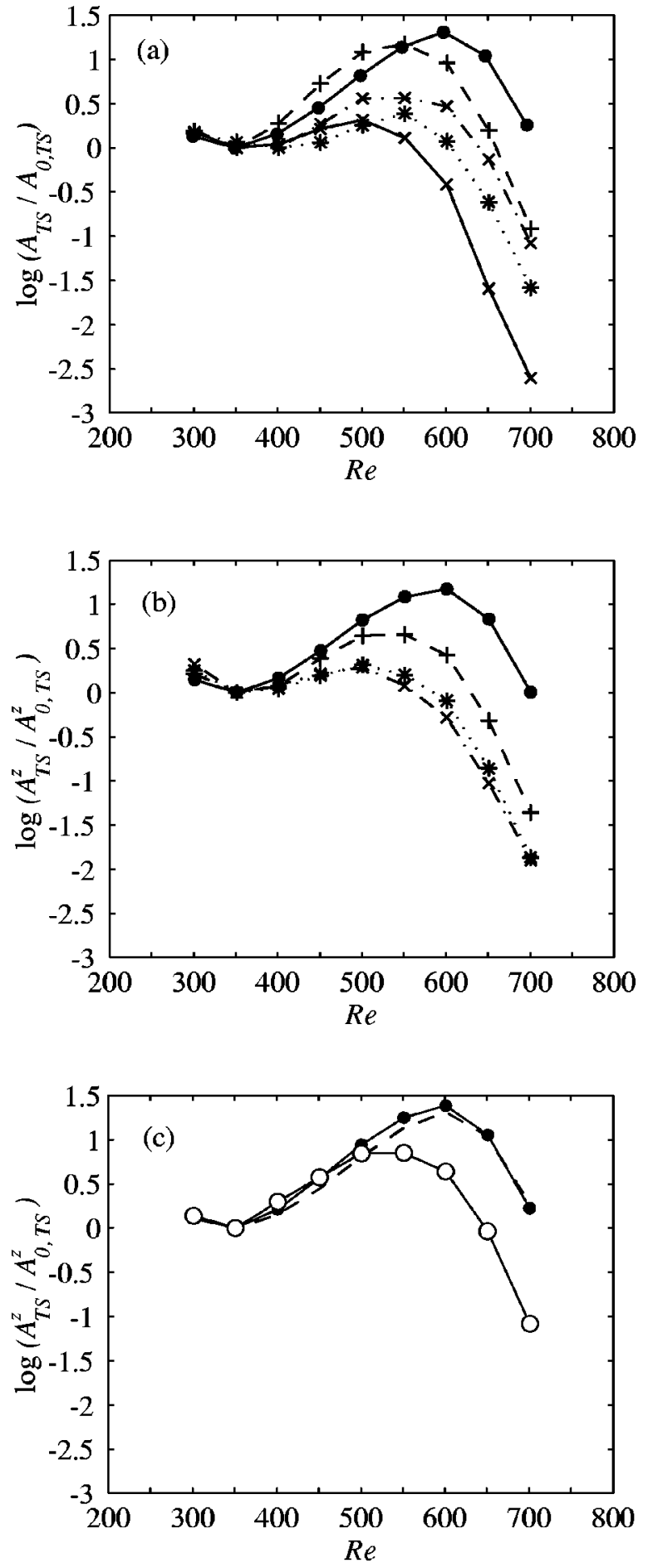

FIG. 18. Alternative amplitude measures. (a) shows the maximum measure, (b) the y-integral measure, and (c) the z-integral measure. The different symbols correspond to $R$ case $(\bullet) ; S_{2}$ case $(+)$ high speed, $(*)$ shear, $(\times)$ low speed, and $(\bigcirc)$ the spanwise integrated. Dash-dotted and solid lines of $(\times)$ correspond to outer and inner peaks, respectively, and other lines are for visual aid only. See text for dashed line in (c). $F=130$.

phase is rapidly changing close to the maximum of the velocity fluctuations profile whereas it is almost independent of $\eta$ in the upper part of the boundary layer. As a consequence, the data at the latter position give more consistent results, basically independent of the spanwise location. The streamwise evolution of the phase pertaining to the five frequencies

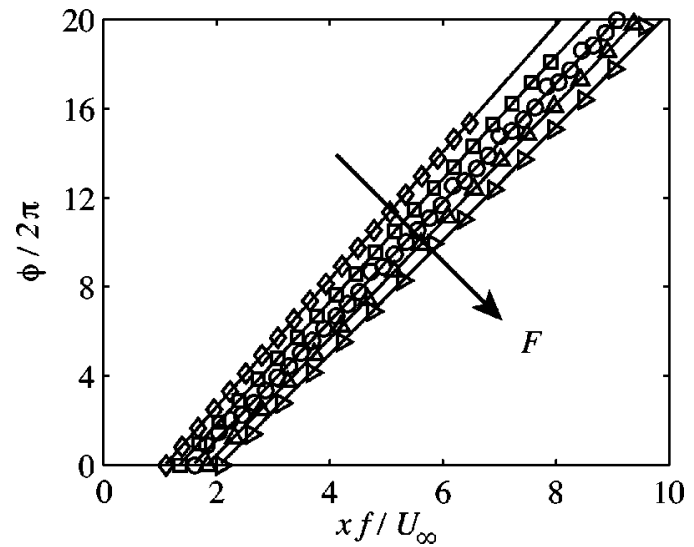

FIG. 19. Streamwise evolution of the phase pertaining to the five frequencies under consideration. The lines represent linear curve fit to the data.

under consideration is shown in Fig. 19, whereas the values of the phase speed extrapolated from the data in the figure are summarized in Table II. It can be noted that the phase speed increases with the frequency $F$. The phase speed of the 2D-TS waves, evolving in the undisturbed boundary layer, can also be found in the Table II. These values, however, are obtained by measuring only close to the wall around the streamwise disturbance maximum which is the standard procedure in a 2D base flow. Furthermore, the values obtained from the linear stability calculations are also reported in Table II where the Re of maximum growth for each frequency (see Figs. 14 and 15) have been used for the theoretical calculated values. This result seems to be in agreement with the predictions by Cossu and $\mathrm{Brandt}^{8}$ that the phase speed is only slightly changed by the presence of streaks.

\section{E. Comparison with theory}

In this section we, first, analyze the difference between the maximum versus the $y$-integral local amplitude measures from a theoretical point of view using linear parallel stability theory (LPT). Second, impulse response analysis and stability calculations are applied to the present experimental 3D streaky base flow enabling a qualitative comparison of the 3D modulated TS wave distribution.

\section{Comparison between different measures}

The range of applicability of parallel stability theory was not ascertained before Klingmann et al. ${ }^{19}$ pursued the conjecture from Saric $^{27}$ that previous stability measurements in Blasius boundary layers were influenced near the minimum critical Re by leading edge pressure gradients, and designed an asymmetric leading edge for their flat plate. The results

TABLE II. Phase speeds of the 2D-TS waves and the $S_{2}$ streaky TS waves at the five frequencies under consideration.

\begin{tabular}{cccccc}
\hline \hline$F$ & 90 & 110 & 130 & 150 & 170 \\
\hline TS waves (Expt.) & 0.38 & 0.36 & 0.38 & 0.39 & 0.35 \\
TS waves (LPT) & 0.35 & 0.36 & 0.37 & 0.38 & 0.38 \\
Streaky TS (Expt.) & 0.35 & 0.36 & 0.38 & 0.38 & 0.39 \\
\hline \hline
\end{tabular}


reported in Ref. 19 showed indeed that the discrepancies with the theory previously observed were due to leading edge effects and thus linear parallel stability theory became well accepted as a tool to predict the evolution of experimentally induced TS waves in a flat plate boundary layer. In the parallel approximation such waves are governed by the coupled Orr-Sommerfeld and Squire equations, and after applying the normal-mode hypothesis, this results in an eigenvalue problem which in a spatial context is solved for the complex streamwise wavenumber $\alpha$ for each desired real frequency (see, e.g., Ref. 28). The real part of $\alpha\left(\alpha_{r}\right)$ together with the angular frequency $\omega$ defines the phase speed of the mode considered whereas the imaginary part $\alpha_{i}$ defines the growth factor.

The relative amplitude ratio $A_{\mathrm{TS}} / A_{\mathrm{TS}, 0}$ defined as

$$
N(\mathrm{Re})=\log \left(A_{\mathrm{TS}} / A_{\mathrm{TS}, 0}\right)=-2 \int_{\mathrm{Re}_{0}}^{\mathrm{Re}} \alpha_{i}\left(\mathrm{Re}^{\prime}\right) d \mathrm{Re}^{\prime}
$$

and computed by solving the Orr-Sommerfeld, Squire system has been shown to agree well with the experimental data in a $2 \mathrm{D}$ base flow if the inner maximum of the streamwise velocity amplitude $A_{\mathrm{TS}}^{\max }$ is considered.

In the undisturbed $2 \mathrm{D}$ case the streamwise eigenfunction $f(\operatorname{Re}, \eta)$ is not self-similar and the $y$-integral measure will differ from the maximum measure. However, since the maximum measure agrees well with the theoretical $N(\operatorname{Re})$ curve [Eq. (5)], one would like to derive an expression for the amplification factor corresponding to this integral amplitude measure based on linear parallel stability theory for a 2D base flow. This amounts to the following expression

$$
A_{\mathrm{TS}}^{y}=\mathcal{C} \cdot \int_{0}^{\eta^{*}} f^{*}(\mathrm{Re}, \eta) \exp [N(\mathrm{Re})] d \eta,
$$

where $f^{*}$ is the eigenfunction normalized to unity and $\mathcal{C}$ $=A_{\mathrm{TS}, 0} / U_{\infty}$ disappears when the relative amplitude ratio is calculated. Since the TS eigenfunction is shifted towards the wall with increasing Re, its total "area" is reduced and a smaller amplification is expected from the integral measure when compared to the maximum measure. This is confirmed in Fig. 20(a) where the two different measures are compared by using both experimental (symbols) and theoretical (lines) data. Here, the theoretical results are computed using the spatial linear stability code presented in the work by Fransson and Alfredsson. ${ }^{29}$ The base flow used in the computations is the FS boundary layer with $m=-0.0046$. It can be seen that the agreement between theoretical and experimental data is found to be good for both amplitude measures. It is useful to note that the higher the truncated value of $\eta^{*}$ is, the larger becomes the difference between the two amplitude measures. In Fig. 20(b) both the mean flow and the TS amplitudes are plotted at three different spanwise positions close to branches I and II. The symbols show excellent agreement with the solid lines, which are the corresponding theoretical profiles.

\section{Linear stability of the streaky base flow}

The temporal stability of the streaky boundary layer flow obtained in the present experimental setup is investigated

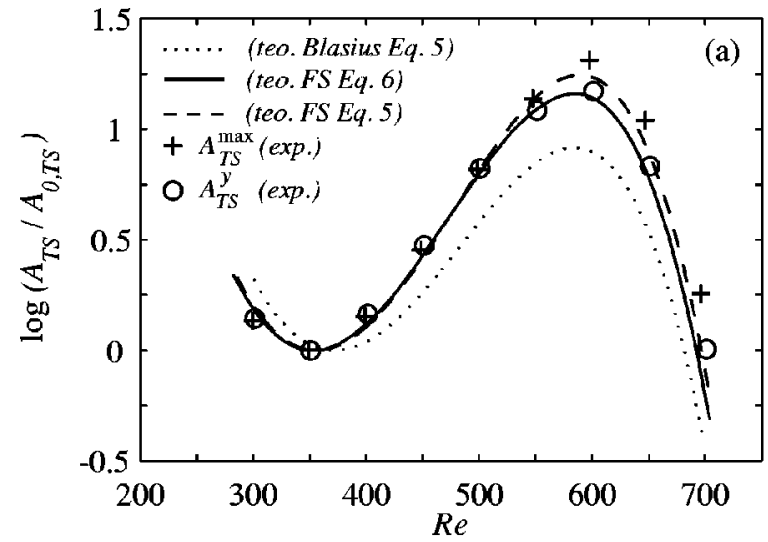

(b)

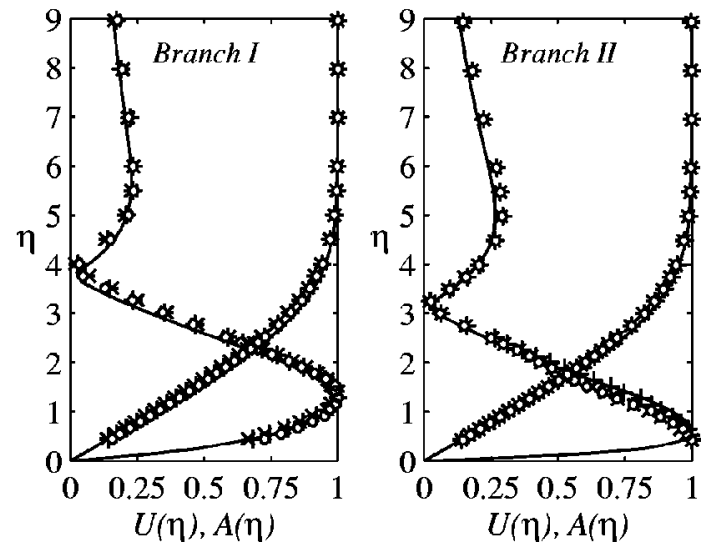

FIG. 20. (a) TS amplification curves of the $R$ case. (b) Shows the wallnormal mean $(U)$ and TS amplitude $(A)$ profiles for three different spanwise positions $(+, \times, \bigcirc)$ close to branches $\mathrm{I}(\mathrm{Re}=351)$ and II $(\mathrm{Re}=601)$. Solid lines are the theoretical FS profiles with $m=-0.0046 . F=130$.

numerically. The approached used is described in the work by Reddy et al. $^{30}$ and in Ref. 8, among others. The numerical code used is the same as in Ref. 30, which allows the calculation of the full spectrum of the stability operator. The results are also verified against the numerical simulation of the flow linear impulse response (see the work by Brandt $e t a l .^{6}$ for a complete description of the numerical method). The base flow considered pertains to case $S_{2}$ at $\operatorname{Re}=650$, where the streak amplitude is about $6 \%$ of $U_{\infty}$. An experimental measurement mesh of $64 \times 41$ points (spanwise and wallnormal directions, respectively) was used as an input to the calculation to represent one spanwise period of the streaky base flow. The experimental data are smoothed and made symmetric and periodic in $z$ in order to be able to perform our analysis on a spanwise periodic base flow as in Ref. 8 . These operations amount to just a small change of the measured data. The results of the linear stability calculations are displayed in Fig. 21 for the fundamental varicose mode. It has in fact been shown in Ref. 8 that the TS waves evolve into varicose fundamental modes in the presence of spanwise modulations of the base flow. The least stable eigenfunction, computed for $\alpha=0.175$, is displayed in Fig. 22. This value of $\alpha$ has been chosen in order to compare the present results with the streamwise velocity fluctuations reported in Ref. 9 since the associated frequency is $F=110$. The agreement be- 

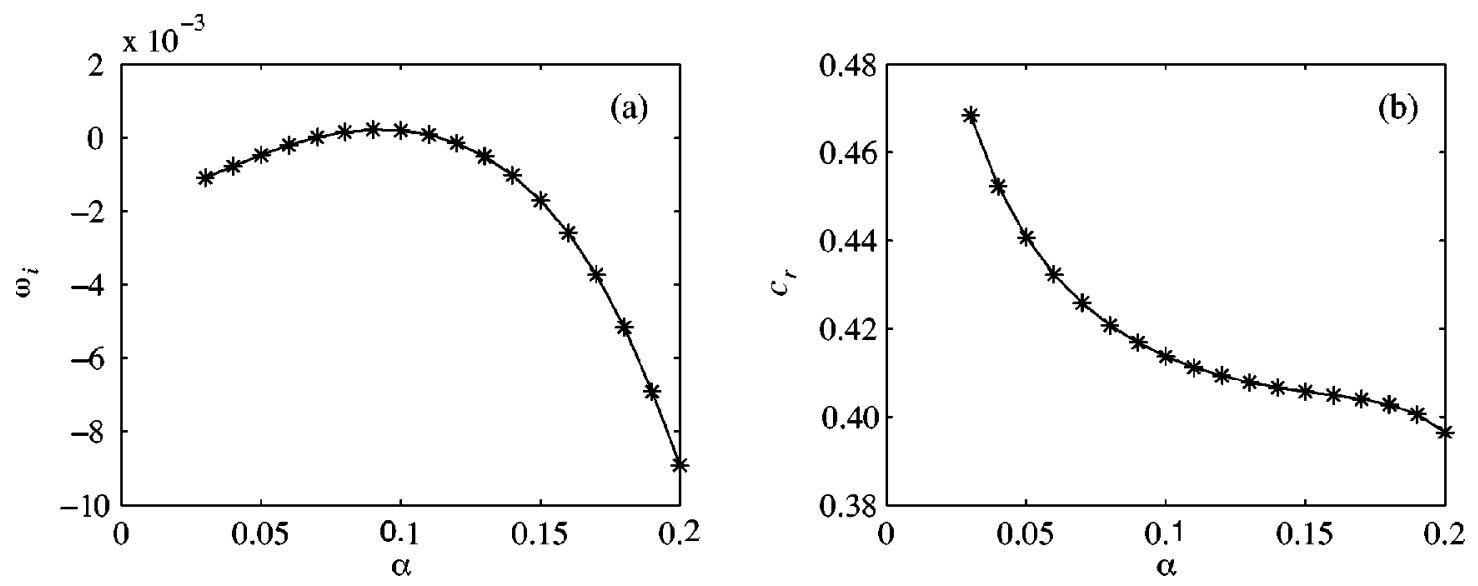

FIG. 21. Temporal stability calculation for the streaky base flow $S_{2}$ at $\operatorname{Re}=650$. (a) temporal growth rate $\omega_{i}$ and (b) phase speed $c_{r}$ vs the streamwise wavenumber $\alpha$.

tween the experimental data can be seen as very good. For this frequency a phase speed $c_{r}=0.36$ is measured. This value is slightly lower than those obtained from the stability calculations [cf. Fig. 21(b)].

\section{CONCLUSIONS}

In this paper we have reported the results of experiments on the stability of Tollmien-Schlichting waves in a boundary layer where moderate amplitude streaks are forced by a spanwise array of roughness elements. For such a moderate amplitude of the streaks previous numerical studies, awaiting full experimental confirmation, predicted that the most unstable Tollmien-Schlichting waves grow less than in the absence of the streaks.

Upon measuring the Tollmien-Schlichting profiles in the full cross-stream plane we have been able to follow the downstream evolution of the TS amplitude by considering the cross-plane integral of its rms velocity $u_{\mathrm{rms}}(y, z)$. This enables us to experimentally verify the stabilizing action of the streaks on the TS waves. By repeating the measures with higher roughness elements, inducing larger amplitude streaks, we are also able to show that the stabilizing action

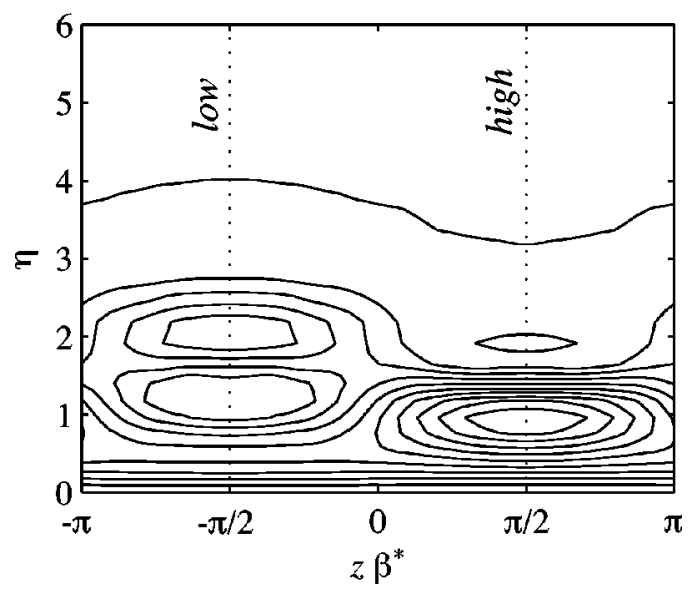

FIG. 22. Theoretical amplitude of the streamwise velocity component for the 3D-TS wave computed at $\operatorname{Re}=650$ for $\alpha=0.175$. Contour levels ranging from 0.1 of the maximum value $\hat{u}_{\max }$ to $0.9 \hat{u}_{\max }$, with spacing $0.1 \hat{u}_{\max }$. on the TS waves increases with the streak amplitude. These results therefore confirm the numerical predictions reported in Refs. 7 and 8.

We considered also the streamwise evolution of the TS wave amplitudes which is obtained when the same amplitude measures used in previous experimental studies are adopted. The results on the present streaks are in qualitative accordance with previous studies ${ }^{14-16}$ where the streaks were induced in a different way. This indicates that the discrepancies between these previous experimental works are probably due to the different definitions of the TS amplitude and not due to differences in the particular generated streaks.

When the amplitude of the TS wave is computed using its wall-normal maximum at a given spanwise position, the TS waves are seen to grow more than in the absence of streaks in the high-speed streak, whereas in the low-speed streaks the perturbation increases less in the presence of the streaks. This is no more true if the wall-normal integral, still at fixed spanwise location, is considered. In this case, in fact, the stabilizing effect of the streaks can be seen both in the high-speed and in the low-speed region. The amplitude measure based on the wall-normal maximum seems therefore too sensitive to the downstream evolution of the shape of the streak. The use of the spanwise integral of the velocity rms at constant distance from the wall ${ }^{15}$ seems to be more appropriate for streaky base flows but it may give different results depending on the wall-normal position where the measurements are considered. The general conclusion that can be drawn from this analysis is that, if possible, the integral of the velocity fluctuation level has to be performed in a cross plane to fairly estimate the growth or decay of perturbations evolving on a streaky base flow. In any case, integral measures should be preferred to measures based on the maximum values.

The 3D waves evolving on the streaks are also documented here and the results are in general agreement with the previous studies. However, it should be noted that mode shape analysis (such as in Fig. 12) can be deceiving and comparisons with other experiments should be treated with care since the TS wave shape solely depends on the 3D modulated base flow and may therefore differ significantly. 
The stabilization achieved in the present study is limited by the streak amplitude. Using cylindrical roughness elements we have not been able to generate stable streaks of amplitude larger than $0.12 U_{\infty}$. This is mainly due to an intrinsic limitation of the generating device. When the Reynolds number $\mathrm{Re}_{k}$ based on roughness element height is larger than about 400, a vortex shedding instability develops in the wake behind the element. This time-periodic shedding induces transition in the otherwise stable streaky boundary layer. At present it is not clear how to overcome this problem but future investigation is granted.

\section{ACKNOWLEDGMENTS}

Financial support from The Swedish Research Council (VR) is greatly acknowledged. The authors wish to thank Professor P. Henrik Alfredsson for all valuable suggestions during the experiments and Marcus Gällstedt for his assistance in the roughness elements manufacturing. A.T. wishes to thank the Italian Ministry of Research MIUR.

${ }^{1}$ H. Schlichting, Boundary-Layer Theory (McGraw-Hill, New York, 1979).

${ }^{2}$ M. T. Landahl, "A note on an algebraic instability of inviscid parallel shear flows," J. Fluid Mech. 98, 243 (1980).

${ }^{3}$ P. Andersson, M. Berggren, and D. S. Henningson, "Optimal disturbances and bypass transition in boundary layers," Phys. Fluids 11, 134 (1999).

${ }^{4} \mathrm{P}$. Luchini, "Reynolds-number independent instability of the boundary layer over a flat surface. Part 2: Optimal perturbations," J. Fluid Mech. 404, 289 (2000)

${ }^{5}$ P. Andersson, L. Brandt, A. Bottaro, and D. S. Henningson, "On the breakdown of boundary layers streaks," J. Fluid Mech. 428, 29 (2001).

${ }^{6}$ L. Brandt, C. Cossu, J. M. Chomaz, P. Huerre, and D. S. Henningson, "On the convectively unstable nature of optimal streaks in boundary layers," J. Fluid Mech. 485, 221 (2003).

${ }^{7}$ C. Cossu and L. Brandt, "Stabilization of Tollmien-Schlichting waves by finite amplitude optimal streaks in the Blasius boundary layer," Phys. Fluids 14, L57 (2002).

${ }^{8}$ C. Cossu and L. Brandt, "On Tollmien-Schlichting-like waves in streaky boundary layers," Eur. J. Mech. B/Fluids 23, 815 (2004).

${ }^{9}$ J. H. M. Fransson, M. Matsubara, and P. H. Alfredsson, "Transition induced by free stream turbulence," J. Fluid Mech. 527, 1 (2005).

${ }^{10}$ M. Matsubara and P. H. Alfredsson, "Disturbance growth in boundary layers subjected to free stream turbulence," J. Fluid Mech. 430, 149 (2001).

${ }^{11}$ K. J. A. Westin, A. V. Boiko, B. G. B. Klingmann, V. V. Kozlov, and P. H. Alfredsson, "Experiments in a boundary layer subject to free-stream turbulence. Part I: Boundary layer structure and receptivity," J. Fluid Mech. 281, 193 (1994)
${ }^{12} \mathrm{D}$. Arnal and J. C. Juillen, "Contribution expérimentale à l'étude de la reptivité d'une couche limite laminaire à la turbulence de l'écoulement général," ONERA, Rapport Technique No. 1/5018, 1978.

${ }^{13}$ A. V. Boiko, K. J. A. Westin, B. G. B. Klingmann, V. V. Kozlov, and P. H. Alfredsson, "Experiments in a boundary layer subjected to free stream turbulence. Part 2. The role of TS-waves in the transition process," J. Fluid Mech. 281, 219 (1994).

${ }^{14}$ I. Tani and H. Komoda, "Boundary layer transition in the presence of streamwise vortices," J. Aerosp. Sci. 29, 440 (1962).

${ }^{15}$ Y. S. Kachanov and O. I. Tararykin, "Experimental investigation of a relaxing boundary layer," Proc. Siberian Div. USSR Acad. Sci., Ser. Tech. Sci. 18(5), 9-19 (in Russian).

${ }^{16}$ A. A. Bakchinov, G. R. Grek, B. G. B. Klingmann, and V. V. Kozlov, "Transition experiments in a boundary layer with embedded streamwise vortices," Phys. Fluids 7, 820 (1995).

${ }^{17}$ J. H. M. Fransson, L. Brandt, A. Talamelli, and C. Cossu, "Experimental and theoretical investigation of the non-modal growth of steady streaks in a flat plate boundary layer," Phys. Fluids 16, 3627 (2004).

${ }^{18}$ B. Lindgren and A. V. Johansson, "Evaluation of the flow quality in the mtl wind-tunnel," Technical Report No. KTH/MEK/TR-02/13-SE, KTH Mechanics, Stockholm, 2002.

${ }^{19}$ B. G. B. Klingmann, A. Boiko, K. J. A. Westin, V. V. Kozlov, and P. H. Alfredsson, "Experiments on the stability of Tollmien-Schlichting waves," Eur. J. Mech. B/Fluids 12, 493 (1993).

${ }^{20}$ J. H. M. Fransson, "Leading edge design process using a comercial flow solver," Exp. Fluids 37, 929 (2004).

${ }^{21}$ A. V. Johansson and P. H. Alfredsson, "On the structure of turbulent channel flow," J. Fluid Mech. 122, 295 (1982).

${ }^{22}$ P. A. Elofsson, Ph.D. thesis, KTH, Department of Mechanics, Stockholm, 1998.

${ }^{23}$ E. B. White and F. G. Ergin, "Receptivity and transient growth of roughness-induced disturbances," AIAA Pap. No. 03-4243 (2003).

${ }^{24}$ M. S. Acarlar and C. R. Smith, "A study of hairpin vortices in a laminar boundary layer. Part 1 . Hairpin vortices generated by a hemisphere protuberance," J. Fluid Mech. 175, 1 (1987).

${ }^{25}$ M. Matsubara, P. H. Alfredsson, and K. J. A. Westin, "Boundary layer transition at high levels of free stream turbulence," ASME Paper 98-GT248 (1998).

${ }^{26}$ H. Fasel and U. Konzelman, "Non-parallel stability of flat-plate boundary layer using the complete Navier-Stokes equations," J. Fluid Mech. 221, 311 (1990).

${ }^{27}$ W. S. Saric, "Low-speed experiments: Requirements for stability measurements," in Instability and Transition, edited by Y. Hussaini (Springer, New York, 1990), Vol. 1, p. 162.

${ }^{28}$ P. J. Schmid and D. S. Henningson, Stability and Transition in Shear Flows (Springer, New York, 2001).

${ }^{29}$ J. H. M. Fransson and P. H. Alfredsson, "On the disturbance growth in an asymptotic suction boundary layer," J. Fluid Mech. 482, 51 (2003).

${ }^{30}$ S. C. Reddy, P. J. Schmid, J. S. Baggett, and D. S. Henningson, "On the stability of streamwise streaks and transition thresholds in plane channel flows," J. Fluid Mech. 365, 269 (1998). 